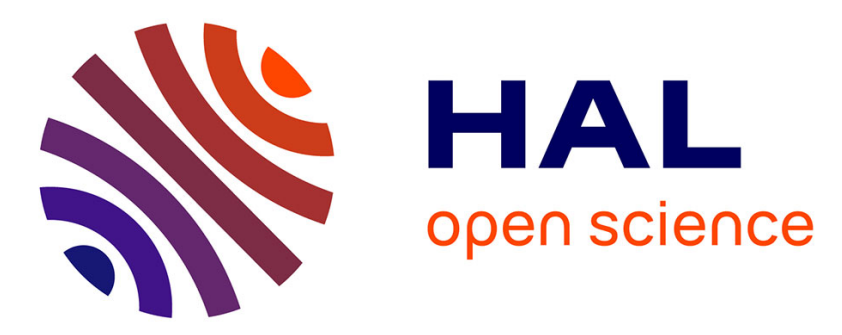

\title{
Counting metastable states within the adsorption/desorption hysteresis loop: A molecular simulation study of confinement in heterogeneous pores
}

\author{
Joël Puibasset
}

\section{- To cite this version:}

Joël Puibasset. Counting metastable states within the adsorption/desorption hysteresis loop: A molecular simulation study of confinement in heterogeneous pores. Journal of Chemical Physics, 2010, 133 (10), pp.104701. 10.1063/1.3483790 . hal-02110061

\section{HAL Id: hal-02110061 https://hal.science/hal-02110061}

Submitted on 26 Nov 2019

HAL is a multi-disciplinary open access archive for the deposit and dissemination of scientific research documents, whether they are published or not. The documents may come from teaching and research institutions in France or abroad, or from public or private research centers.
L'archive ouverte pluridisciplinaire HAL, est destinée au dépôt et à la diffusion de documents scientifiques de niveau recherche, publiés ou non, émanant des établissements d'enseignement et de recherche français ou étrangers, des laboratoires publics ou privés. 


\title{
Counting metastable states within the adsorption / desorption hysteresis
}

\section{loop: a molecular simulation study of confinement in heterogeneous pores}

\author{
Joël Puibasset
}

Centre de Recherche sur la Matière Divisée, CNRS-Université d'Orléans, 1b rue de la Férollerie, 45071 Orléans cedex 02, France

puibasset@cnrs-orleans.fr

\begin{abstract}
A molecular simulation approach has been used to model simple fluid adsorption in heterogeneous tubular pores mimicking mesoporous materials like MCM-41 or porous silicon, allowing to determine the amount adsorbed $\rho$ as a function of the chemical potential $\mu$. A hysteresis loop is observed in adsorption / desorption cycles, which is closely connected to the appearance of many metastable states. The density of these metastable states is studied in the $\mu-\rho$ plane. Experimentally, the accessible metastable states are those that can be attained by $\mu$-path, i.e. a series of increasing or decreasing $\mu$ steps. One could also imagine using a quench from high temperature. Although the total density of metastable states is not directly accessible to experiments, it is of primary theoretical importance to understand the
\end{abstract}


structure of metastable states in the hysteresis as determined experimentally. The disorder associated to the porous material realizations is accurately taken into account, and a systematic system-size analysis is also performed in order to study the thermodynamic limit. It is shown that the quenched complexity is the relevant quantity to understand the hysteresis structure in the thermodynamic limit. It clearly exhibits a distinctive behavior depending on the distribution of heterogeneities characterizing the disorder in the pore. Some analogies can be found with the situation where an out-of-equilibrium transition appears, but careful examination of the data suggests another interpretation.

\section{Introduction}

Despite its apparent simplicity, adsorption of fluids in regular mesoporous solids is still challenging. Many experimental efforts have been made to produce such solids presenting almost perfectly cylindrical and non-interconnected mesopores, like MCM-41, ${ }^{1,2}$ and porous silicon. ${ }^{3}$ Due to their importance for application and fundamental studies, they have been widely characterized, and many experimental efforts have produced accurate measurements of adsorption/desorption hysteresis in such materials for a large variety of fluids. ${ }^{4-9}$ Extensive theoretical work has provided deep understanding of many issues, but some points remain unclear even for the above mentioned regular mesopores. ${ }^{10}$

One of the puzzling behaviors is the appearance of large hysteresis in regular mesopores even when they are closed at one end. ${ }^{7,11}$ Wallacher and collaborators ${ }^{8}$ have performed extensive studies showing that the pores of porous silicon most probably present an intrinsic disorder which is more efficient to produce hysteresis than the enforced modulation of the pore size 
during the material production. Other authors arrived at the same conclusion that the inner surfaces of MCM-41 or porous silicon most probably exhibit some roughness and irregularity. ${ }^{8,12-18}$

The quenched or frozen disorder, originating in the irregular structure of the solid or the solidfluid interaction, has been shown to explain the hysteresis in the case of gas adsorption in 3dimensional structures like aerogels. ${ }^{19-21}$ It was however not yet clear if such disorder could explain hysteresis in non-interconnected mesopores like MCM-41 or porous silicon (1dimensional pore structure). To answer the question, we focused on a molecular approach to catch the main features of fluid adsorption. We introduced a simplified model of surface heterogeneities based on preliminary studies showing that the modulation of the fluid/wall interaction has strong consequences on fluid adsorption in simple pore geometry, while purely geometric deformation of the pore without changing the intensity of the fluid/wall potential has minor effects. ${ }^{22-25}$ Note that a constriction in a pore is generally associated to both a geometric deformation and a modulation of the fluid/wall interaction (the smaller the diameter of the constriction, the larger the local fluid/wall interaction), and thus plays an important role in gas adsorption or meniscus displacement. ${ }^{26,27}$ Simple models of inhomogeneous linear pores could then be built by modulating the fluid/wall potential along the cylindrical pore axis. Molecular simulations in the grand canonical ensemble allowed to study the cases of few heterogeneities, or domains as introduced by Everett. ${ }^{28}$ New metastable states where shown to be stabilized by the most attractive portions of the pore, and complex coexistence diagrams of the various metastable states were proposed. ${ }^{29}$ The complexity is growing faster than the number of domains, and the time-scale of the simulation did not allow extensive studies on larger systems. A multiscale approach could however be introduced because it is possible to study each domain of a tubular pore if one knows the fluid state in the two neighboring 
domains (the pores are non-interconnected, and thus present a 1D chain structure). This approach allowed to study pores of micrometric length, presenting few hundred domains, much beyond computer capabilities for a brute force molecular simulation. It is emphasized that this approach completely differs from pore network approaches where simple rules are postulated for filling and emptying (pore blocking etc.). ${ }^{30}$ Within this approach it has been shown that the presence of disorder in tubular pores induces the appearance of a large hysteresis in adsorption/desorption cycles, very similar to what is experimentally observed in disordered materials like aerogel or Vycor. ${ }^{31}$ This behavior arises in each pore, as a consequence of disorder, and does not require any connection between pores. Naumov and co-workers arrived at similar conclusions in a simplified lattice gas approach. ${ }^{32}$ The effect of an additional disorder between pores (like a pore size distribution) has also been considered and shown to be negligible when the pores already present an intrinsic disorder. ${ }^{33}$

The main scanning curves obtained in the grand canonical ensemble, i.e. desorption or adsorption branches started from any given point of the main hysteresis loop, are qualitatively comparable to experimental data: they reveal the presence of a large number of metastable states which densely fill up the whole hysteresis since all scanning curves are continuous and can be close parallel for slightly different initial filling of the porous material. Studying these curves constitutes a preliminary exploration of the underlying organization of the metastable states which might contain some information on the mesoporous solid structure. However, only few metastable states can be attained by first scanning curves, ${ }^{34}$ and a good understanding of hysteresis requires a more systematic search for all metastable states. Such studies are generally extremely difficult, especially if one wants to infer conclusions in the thermodynamic limit, i.e. the experimental situation. Such questions have been slightly more 
studied in other fields like hysteresis in magnetic materials, but recent works have shown that their relevance to adsorption hysteresis cannot be underestimated. ${ }^{35,36}$

As shown in previous papers, the fluid may be in essentially two states in each domain. This allows a systematic study of all possible states of the confined fluid, at least for small enough systems. An algorithm (given below) determines which states are metastable and their location in the $\mu-\rho$ plane (chemical potential versus amount adsorbed). However, due to the exponential increase of the number of possible states with the number of domains, the study is limited to quite small systems with typically less than 35 domains. Two issues need then to be addressed: 1) the results actually strongly depend on the particular distribution of heterogeneities in the porous material. One thus has to somehow perform an average over disorder, i.e. over many possible pore samples or "realizations". 2) the thermodynamic limit of infinitely long pore has to be discussed. These issues cannot be addressed rigorously in our numerical approach, except when exact calculations are possible (used as benchmark). Reasonable approximations allow however to determine appropriate methods to perform average over disorder, and a systematic finite size analysis allows to infer trends for the thermodynamic limit. Note that in a typical MCM-41, pores are several micrometers long, corresponding to approximately $10^{2}-10^{3}$ domains. The thermodynamic limit might thus not be the only relevant case. In this first study of the metastable state structure we focus on their density distribution inside the hysteresis loop, and the associated complexity, i.e. the logarithm of the density normalized to the system size. Two examples are studied, one with usual continuous adsorption/desorption isotherms, and one exhibiting a sharp jump during adsorption. They are shown to present important differences in their distribution of metastable states, suggesting the existence of an underlying transition. 
The paper is organized as follows: In section II, we introduce the pore model, the algorithms used to determine the isotherms and metastable states, and briefly introduce general results regarding disordered systems, in particular the annealed and quenched averages, where the variables associated to the source of disorder, originating in the pore irregularities in our case, are either free to fluctuate or frozen. In section III we present the isotherms and all metastable states for various pore samples. The distributions of the number of metastable sates over disorder realizations and for a given chemical potential are shown and discussed. The quenched complexity is shown to appear as self-averaging. In section IV and V we properly define and calculate, in finite size, the annealed and quenched complexities as a function of the chemical potential $\mu$, and as a function of $\mu$ and the amount adsorbed $\rho$. Finally, in section VI, the case of a porous material exhibiting a sharp jump during adsorption is studied. We finally conclude by comparing the two systems and the qualitative differences observed for the quenched complexity.

\section{Model and methods}

Our model is designed to catch the main features of simple fluid adsorption in heterogeneous tubular pores like MCM-41 or oxidized porous silicon. The pores, of diameter around $3 \mathrm{~nm}$, are supposed to be made of pure silica. The interactions are supposed to follow the LennardJones potential. The parameters are those for argon-argon and argon-oxygen species to describe fluid-fluid and fluid-silica interactions. ${ }^{31}$ All thermodynamic quantities are reduced using fluid-fluid interaction parameters. Heterogeneities are introduced by modulation of the fluid/wall interaction along the pore axis, within $+/-30 \%$. The modulation factor, denoted $h$, is the ratio between the local fluid/wall interaction intensity to its reference value. For 
instance, $h=0.85$ means that the interaction is 15 percent lower. It is chosen to be a step function along the pore axis. Each step corresponds to a domain as defined by Everett ${ }^{28}$ of approximately $7 \mathrm{~nm}$. The values of the steps are uncorrelated and follow a Gaussian distribution centered on 1.0 and half-width 0.2 in the interval $[0.7 ; 1.3]$. Interactions being short range, typically less than a domain length, the fluid properties in a given domain are entirely determined by the fluid configurations in the two neighboring domains. Since fluidfluid correlations decrease very rapidly, the relevant quantity in the neighboring domains is, on average, the fluid density. This is entirely determined by the chemical potential $\mu$, except if the adsorbed fluid may exist in several states. This is precisely the case for a fluid confined in a tubular domain, which, for some values of $\mu$, may be in the gaslike $(\mathrm{G})$ or liquidlike (L) state, corresponding respectively to fluid adsorbed only at the wall or filling the whole domain. As a consequence, the fluid properties in a given domain and for a given $\mu$ are entirely determined by the fluid state ( $\mathrm{G}$ or $\mathrm{L}$ ) in the two neighboring domains. At the most 4 possibilities need to be considered. Figure 1 shows a typical set of 4 such "conditional" isotherms obtained in a cylindrical domain. After these calculations are performed for all possible domains, the fluid properties can be easily determined in the whole pore, according to the algorithms described below. This approach allows a decoupling between the atomistic level calculations performed by Grand Canonical Monte Carlo molecular simulation in each domain, and the large-scale calculation of the fluid state in pores of micrometric length. More details on the model can be found in refs $\left[{ }^{31}\right]$ and $\left[{ }^{33}\right]$. With this approach we are able to study fluid properties in very long pores at the atomistic level, or perform extensive studies like counting metastable states in systems of reasonable sizes, which is impossible otherwise. In the rest of the paper, we consider only pores open at both ends: as a consequence, the first and last domains are always in contact with the bulk gas state. 
The adsorption/desorption isotherms in a complete pore are calculated as follows: For low $\mu$, the adsorption in each domain is single valued, and does not depend on the fluid state in the rest of the pore. The amount adsorbed is simply the sum over all domains. Note that in this case, all domains are in the gaslike state and follow the conditional isotherm with G-G boundaries. The chemical potential is then increased by small amounts until one domain reaches its limit of stability. This domain then jumps to the liquidlike branch. This has consequences for the two neighboring domains, which boundaries are now G-L and L-G. Their state has then to be reconsidered according to their G-L isotherms. They may stay in the gaslike state, meaning that the fluid condenses only in the initial domain, or switch to the liquidlike state, meaning that the meniscus created in the initial domain propagates to neighboring domains. In this case, the next domains need now to be reconsidered too, and so on until the meniscus stops, corresponding to the end of the avalanche. The amount adsorbed is given by the sum over all domains. The chemical potential is then further increased, and the same procedure is applied until saturation is reached. Desorption and scanning isotherms are obtained in a similar way.

We now present the method used to determine all metastable states for any given realization of the disorder in the pore and for a given chemical potential $\mu$. As previously explained, the fluid properties are entirely determined by the chemical potential $\mu$ and the fluid state (G or L) in each domain. One then expects at the most $2^{N}$ possible fluid states, where $N$ is the number of domains. However, due to the interdependence between neighboring domains, many configurations are actually impossible. For instance, a domain in between a gaslike and a liquidlike domain, actually has only one (instead of two) possible state entirely determined by the chemical potential since G-L isotherms are single valued. But many other situations may appear, depending on the exact forms of the pre-calculated isotherms. From a physical 
viewpoint, these configurations correspond to situations where the fluid state is unstable and has to evolve towards another state, by filling or emptying some domains. On the other hand, the configurations which are self-consistent are stable against thermal fluctuations, which are taken into account in the pre-calculated isotherms, and correspond to the metastable states. For any given $\mu$, the algorithm explores all possible states, and extracts the few ones that are self-consistent, i.e. metastable. It is observed that any given metastable state can exist on a range of $\mu$, which can easily be determined by slowly decreasing (increasing) $\mu$ until the lower (upper) stability limits $\mu_{\min }\left(\mu_{\max }\right)$ are reached. The corresponding $\rho(\mu)$ curves can be drawn in the $\mu-\rho$ plane to represent each metastable state.

The number of possible fluid states to be examined increases exponentially with system size. As a consequence, our algorithm cannot consider pores with more than 35 domains for few disorder realizations, 30 domains for $5 \times 10^{3}$ disorder realizations, 25 domains for $5 \times 10^{4}$ disorder realizations, and 20 domains or less for $10^{5}$ disorder realizations. It will however be shown below that the results indicate that the thermodynamic limit can be reasonably extrapolated from these system sizes. The last difficulty is how to perform averages over disorder realizations? Each realization produces its own hysteresis. How could we manage to get an "average" hysteresis? For a given chemical potential far from the hysteresis region, the fluid state is single-valued and given by the amount of fluid adsorbed. This quantity being additive, one expects that the "average" fluid state over disorder be precisely the averaged amount adsorbed. Note that the additivity hypothesis relies on the fact that for large enough systems, the amount adsorbed in any macroscopic region of the porous solid is not very different from what would be obtained if this region was isolated from the rest of the material. However, in the hysteresis region, the amount adsorbed is not uniquely defined, and can take any value between the so-called main adsorption and desorption branches. One can easily 
average these main branches over disorder to define the "average main hysteresis loop". However, characterizing the distribution of metastable states within the hysteresis and performing the average is a much more difficult task, to be explained now.

Let us define the density of metastable states in the $\mu$ - $\rho$ plane $\mathcal{N}(\rho ; \mu ; N)$, where $N$ is the number of domains in the pore. By definition $\mathcal{N}(\rho ; \mu ; N) d \rho$ is, for a given chemical potential $\mu$, the number of metastable states with adsorbed density in the interval $d \rho$ around $\rho$. Note that this definition of $\mathcal{N}(\rho ; \mu ; N)$ as a density is necessary in our case (continuous values for $\rho$ ) and differs slightly from the usual definition used in Ising models where $\rho$ is discrete. This has no consequences for the definition of the corresponding complexities (see Eqs. 1 and 2 below) since the differences vanish in the thermodynamic limit. We also define the number of metastable states at a given $\mu$ as $\mathcal{N}(\mu ; N)=\int \mathcal{N}(\rho ; \mu ; N) d \rho$. The total number of metastable states is denoted $\mathcal{N}(N)$. Note that despite similar notation " $\mathcal{N}$ ", these quantities are not identical and the parentheses will help to differentiate them. Furthermore, one has to keep in mind that $\mathcal{N}(N)$ is not the integral of $\mathcal{N}(\mu ; N)$ over $\mu$ because all metastable states exist over a finite range of $\mu$, and thus each metastable state would be counted several times.

The density of metastable states $\mathcal{N}(\rho ; \mu ; N)$ not only depends on the statistical properties of the disorder in the pore, but is also strongly sensitive to the particular disorder realization in the pore sample. It is then natural to introduce an average over disorder. As usual in disordered systems, it is interesting to consider both the average and typical number of metastable states: $\overline{\mathcal{N}(\rho ; \mu ; N)}$ and $\exp [\overline{\ln \mathcal{N}(\rho ; \mu ; N)}]$ where the bar denotes the average 
over disorder. ${ }^{35,36}$ Since these quantities are both expected to scale exponentially with $N$, one introduces the corresponding annealed and quenched complexities:

$$
\begin{aligned}
& \sum_{\mathrm{A}}^{\infty}(\rho ; \mu)=\lim _{N \rightarrow \infty} \frac{1}{N} \ln \overline{\mathcal{N}(\rho ; \mu ; N)} \\
& \sum_{\mathrm{Q}}^{\infty}(\rho ; \mu)=\lim _{N \rightarrow \infty} \frac{1}{N} \overline{\ln \mathcal{N}(\rho ; \mu ; N)}
\end{aligned}
$$

As will be shown in next section, the quenched complexity is of particular interest because it is expected to be self-averaging (its fluctuations scale as $1 / \sqrt{N}$ ), which is not the case for the annealed complexity.

\section{Isotherms and metastable states}

The main adsorption/desorption isotherms and all metastable states are calculated for various pore length and disorder realizations. Some examples are shown in Fig. 2 for $N=5,10$ and 15. All isotherms present two reversible portions at low and high $\mu$, and a hysteresis in between. The saturation branch is essentially identical in all cases, whereas the initial adsorption branch exhibits some differences which reflect the variations in the average fluid/wall interaction in the whole pore. These variations are expected to decrease with the system size. As can be seen, the hysteresis is also strongly dependent on the particular realization of the disorder. One can notice differences in width, height, and slope of the adsorption and desorption branches.

The metastable states appear as portions of lines corresponding to their metastability range. These lines can be seen as the projection in the $\mu-\rho$ plane of the local minima in the free energy landscape. As expected, the slopes are always positive due to increasing film thickness 
with $\mu$. These lines are almost straight and essentially parallel, meaning that despite possibly large differences in fluid configuration between two adjacent metastable states, the response of the system to a chemical potential variation is essentially the same in both cases. The small difference appears as a balance between the number of domains in the gaslike and the liquidlike state, expected to be of order $1 / N$. However, large variations can be observed in their distribution in the $\mu-\rho$ plane depending on the disorder realization. Nevertheless, in all cases, all metastable states fall within the main hysteresis loop. It is actually observed that the main hysteresis constitutes the external envelope of the metastable states. This feature is observed in other systems and seems to be quite general. ${ }^{35,36}$.

Another interesting quantity is the total number of metastable states $\mathcal{N}(N)$, given in each panel of Fig. 2. The first point to be noticed is that this number grows faster than $N$, but is significantly smaller than the total number of fluid states $2^{N}$, especially for large $N$. It also takes a wide range of values depending on the pore disorder realization. Its distribution has been numerically investigated for various $N$. As can be seen in Fig. 3 (first and second panels) the distributions are quite large, with long tails. As previously noticed in undulated pores, ${ }^{29}$ the typical number of metastable states grows quite fast with the number of domains. This is why the distributions cannot be drawn on the same panel. The average and standard deviation were also calculated for $N \leq 30$, and shown to be compatible with an exponential behavior. Furthermore the width of the distribution remains of the same order as its average value. On the other hand, the distributions for $\ln \mathcal{N}(N)$ are very close to Gaussians (see Fig. 3, third panel). The average value over disorder $\overline{\ln \mathcal{N}(N)}$ is linear with $N$, and the standard deviation of $N^{-1} \overline{\ln \mathcal{N}(N)}$ behaves as $N^{-1 / 2}$. As a consequence, the quenched complexity appears as the natural self-averaging quantity to characterize the number of metastable states. 


\section{Calculation of $\ln \overline{\mathcal{N}(\mu ; N)}$ and $\overline{\ln \mathcal{N}(\mu ; N)}$}

We now focus on the number of metastable states for a given chemical potential $\mu$. Since numerical simulations are performed in finite systems, we define finite size annealed and quenched complexities for a given $\mu$ as:

$$
\begin{aligned}
& \Sigma_{\mathrm{A}}(\mu, N)=\frac{1}{N} \ln \overline{\mathcal{N}(\mu ; N)} \\
& \Sigma_{\mathrm{Q}}(\mu, N)=\frac{1}{N} \overline{\ln \mathcal{N}(\mu ; N)}
\end{aligned}
$$

where $N$ is the pore length. Their corresponding thermodynamic limit $(N \rightarrow \infty)$ are denoted as $\sum_{\mathrm{A}}^{\infty}(\mu)$ and $\sum_{\mathrm{Q}}^{\infty}(\mu)$. Let us first focus on the particular choice $\mu=-8.55$ corresponding to the central region of the hysteresis (see Fig. 2). The finite size complexities have been calculated for $N=15,20,25$ and 30 with respectively $10^{5}, 10^{5}, 5 \times 10^{4}$, and $5 \times 10^{3}$ disorder realizations (circles in Fig. 4). The data exhibit a small dependence with the system size, which is well described by the ansatz $\sum_{\mathrm{A} / \mathrm{Q}}(\mu, N)=\sum_{\mathrm{A} / \mathrm{Q}}^{\infty}(\mu)+a_{\mathrm{A} / \mathrm{Q}}(\mu) N^{-1} \ln N+b_{\mathrm{A} / \mathrm{Q}}(\mu) N^{-1}$ involving three free parameters which depend on $\mu .{ }^{36}$ A least-squares fit to the data for $\mu=-$ 8.55 yields $\sum_{\mathrm{A}}^{\infty}=0.2555, a_{\mathrm{A}}=0.0313, b_{\mathrm{A}}=-7.538 \times 10^{-3}$ and $\sum_{\mathrm{Q}}^{\infty}=0.2266, a_{\mathrm{Q}}=-0.0120$, $b_{\mathrm{Q}}=0.0735$ (the best fit are shown as dashed lines in Fig. 4).

For the particular choice $\mu=-8.55$ an analytical calculation is possible and can be used to check the accuracy of the previous system-size dependence analysis (see Appendix A). The results are shown as red solid lines in Fig. 4. As can be seen, the data obtained by exact enumeration of all metastable states are in close agreement with the expected values, showing 
the good convergence in terms of average over disorder. We are now in a position to address the thermodynamic limit. One can first perform the numerical calculations for the largest possible value of $N$, which gives good results due to the weak dependence with $N$, but the safest is to perform the analytical derivation (see Appendix B). The results are $\sum_{\mathrm{A}}^{\infty}(\mu=-8.55)=0.25715$ and $\sum_{\mathrm{Q}}^{\infty}(\mu=-8.55)=0.22557$. They are given as horizontal straight lines in Fig. 4. These exact calculations show that the numerical finite size analysis was correct within $1 \%$, and is likely to be reliable even when analytical calculations are not possible.

This systematic enumeration of metastable states has been extended to other values of the chemical potential. The results are displayed in Fig. 5 (symbols). The previous theoretical derivation of the annealed and quenched complexities can be extended to any $\mu<-8.53$ (see Appendices). The corresponding theoretical thermodynamic limits are given as lines in Fig. 5. As can be seen, the simulation results exhibit a weak dependence with system size and are quite reliable, even in regions where the complexities vary rapidly. The data show that both complexities are not zero for $\mu$ between approximately -8.67 and -8.25 , and exhibit a maximum around $\mu=-8.58$. As expected, $\sum_{\mathrm{Q}}^{\infty}(\mu) \leq \sum_{\mathrm{A}}^{\infty}(\mu)$. A remarkable feature is that both complexities reach zero for the same chemical potential. It corresponds to the situation where all domains are of the same type. The theoretical derivation shows that both complexities reach zero when the probability $p_{\mathrm{A}}$ reaches zero at $\mu=-8.6757$ : straightforward calculations show that at first order $\sum_{\mathrm{A}}^{\infty}(\mu) \approx p_{\mathrm{A}}(\mu)\left(\lambda_{1}-1\right)$ and $\sum_{\mathrm{Q}}^{\infty}(\mu) \approx p_{\mathrm{A}}(\mu) \ln \lambda_{1}$, for $p_{\mathrm{A}}(\mu) \rightarrow 0$. A similar behavior is expected around $\mu=-8.25$. Note that these points correspond to the lower and upper closure points of the main hysteresis loop. The same is observed in Ising chain models. $^{35}$ 


\section{Calculation of $\ln \overline{\mathcal{N}(\rho ; \mu ; N)}$ and $\overline{\ln \mathcal{N}(\rho ; \mu ; N)}$}

As previously, we introduce the annealed and quenched complexities for finite systems. The annealed complexity associated to the distribution of metastable states versus $\rho$ and $\mu$ is defined as follows:

$$
\Sigma_{\mathrm{A}}(\rho ; \mu, N)=\frac{1}{N} \ln \overline{\mathcal{N}(\rho ; \mu ; N)}
$$

where the bar denotes average over disorder. This quantity has been calculated on a discrete grid in the $\mu$ - $\rho$ plane, for the same $\mu$-values as previously, and for $24 \rho$-values between 0 and 1. Our definition of the complexities based on the density of metastable states thus differs from the one used in Ising models by $-N^{-1} \ln \delta \rho$ where the bin width $\delta \rho=1 / 24$ in our case. Figure 6 shows the results for $N=25$. This system size allows a good compromise between large $N$ and a large number of disorder realizations $\left(5 \times 10^{4}\right)$. For comparison, the average over disorder of the main hysteresis loop for $N=25$ has been superimposed in the top view. As can be seen, the annealed complexity presents a typical shape with maximal values within the region of hysteresis. It can also be noted that the region where the annealed complexity is positive extends well beyond the main adsorption/desorption loop. It also exhibits a spurious finite region where a single fluid state is expected whatever the disorder (for instance for $\mu<-$ 8.7 and $\mu>-8.2$ ). In between these values, visual inspection shows that the hysteresis defined by the main loop qualitatively corresponds to $\Sigma_{\mathrm{A}}(\rho ; \mu, N) \geq-N^{-1} \ln \delta \rho$ (green to red region in Fig. 6), i.e. to an average number of metastable states larger than 1 per bin. How does this change in the thermodynamic limit? The system size dependence is illustrated in Fig. 7 for $\mu$ $=-8.55$. The first point to be noticed is that the system size dependence is significantly more 
pronounced for $\Sigma_{\mathrm{A}}(\rho ; \mu, N)$ than for $\Sigma_{\mathrm{A}}(\mu ; N)$ : symbols are superimposed in Fig. 5, not in Fig. 7. However, as previously, it is possible to extrapolate the thermodynamic limit using the $N$-dependent ansatz $\sum+a N^{-1} \ln N+b N^{-1}$, where the three parameters depend on $\rho$ and $\mu$. When performed for the maxima, occurring around $\rho=0.53$ for $\mu=-8.55$, one gets a limiting value $\Sigma=0.256$ very close to the previously calculated $\sum_{\mathrm{A}}^{\infty}(\mu=-8.55)=0.25715$. This is expected because $\overline{\mathcal{N}(\rho ; \mu ; N)}$ is exponentially large in the thermodynamic limit and thus its integral over $\rho$, giving $\sum_{\mathrm{A}}^{\infty}(\mu)$, is dominated by its largest term. A thermodynamic limit curve can thus be proposed (Fig. 7, dashed line) exploiting the system size dependence. In particular, one observes that the densities for which $\Sigma_{\mathrm{A}}(\rho ; \mu=-8.55, N)=0$ are essentially stable with $N$. Note that, in the thermodynamic limit, the regions defined by $\Sigma_{\mathrm{A}}(\rho ; \mu, N) \geq-N^{-1} \ln \delta \rho$ or $\Sigma_{\mathrm{A}}(\rho ; \mu, N) \geq 0$ are identical and clearly extend well beyond the hysteresis defined by the main adsorption/desorption loop. This conclusion is supported by theoretical works. ${ }^{35,36}$

The finite-size quenched complexity associated to the distribution of metastable states versus $\rho$ and $\mu$ may be introduced as follows:

$$
\sum_{\mathrm{Q}}^{\mathrm{Ch}}(\rho ; \mu ; N)=\frac{1}{N} \overline{\ln \mathcal{N}(\rho ; \mu ; N)}
$$

where the bar denotes average over disorder, and the logarithm is modified as proposed by Cherrier and collaborators: ${ }^{37}$ since for finite systems, the number of metastable states can locally be equal to zero, the logarithm diverges. It is thus arbitrarily set to zero in such situation. This introduces non-controllable errors in the evaluation of the quenched complexity. However, the results are expected to be reliable in the central region of the hysteresis where the density of metastable states almost never vanishes. The results are shown 
in Fig. 8 (for $N=25$ ) as a function of $\rho$ and $\mu$, for $\sum_{\mathrm{Q}}^{\mathrm{Ch}}(\rho ; \mu ; N)>-N^{-1} \ln \delta \rho$ so that the typical number of metastable states is always larger than 1 per bin. The average main hysteresis loop for $N=25$ has been superimposed in the top view. As can be seen, the largest values for the quenched complexity are reached within the hysteresis loop, but, as previously, the region determined by $\sum_{\mathrm{Q}}^{\mathrm{Ch}}(\rho ; \mu ; N)>-N^{-1} \ln \delta \rho$ extends beyond the main adsorption/desorption loop. Note however a significant qualitative improvement for $\mu<-8.68$ and $\mu>-8.28$. The system size dependence is illustrated in Fig. 9 for $\mu=-8.55$. As previously, a best fit can be obtained with the ansatz $\sum+a N^{-1} \ln N+b N^{-1}$, and the limiting value of the maximum for $\mu=-8.55$ is compatible with $\sum_{\mathrm{Q}}^{\infty}(\mu=-8.55)$. A putative thermodynamic limit can be drawn (Fig. 9, dashed line). The figures show that the quenched complexity reaches its largest values inside the hysteresis in the thermodynamic limit. However, since $\sum_{\mathrm{Q}}^{\mathrm{Ch}}(\rho ; \mu ; N)$ is not reliable when it approaches zero, it is impossible to discuss whether the quenched complexity exactly vanishes on the main adsorption/desorption loop in the thermodynamic limit. Another approach is required.

To circumvent the difficulty raised previously, it has been proposed ${ }^{36}$ to introduce the Legendre-Fenchel transform $\Lambda^{\alpha}(g ; \mu ; N)$ of the finite size, disorder dependent complexity

$$
\begin{aligned}
& N^{-1} \ln \mathcal{N}^{\alpha}(\rho ; \mu ; N): \\
& \Lambda^{\alpha}(g ; \mu ; N)=\max _{\rho}\left\{\frac{1}{N} \ln \mathcal{N}^{\alpha}(\rho ; \mu ; N)+g \rho\right\}
\end{aligned}
$$

where $\alpha$ denotes a given disorder realization. Note that this quantity is always defined for any real value $g$, even if $\mathcal{N}^{\alpha}(\rho ; \mu ; N)$ is zero for some values of $\rho$. To see the connection with 
the quenched complexity, let us denote $\rho^{\alpha}(g ; \mu ; N)$ the $\rho$-value which maximizes the quantity between parentheses in Eq. 7. One has:

$$
\Lambda^{\alpha}(g ; \mu ; N)=\frac{1}{N} \ln \mathcal{N}^{\alpha}\left(\rho^{\alpha} ; \mu ; N\right)+g \rho^{\alpha}
$$

Averaging over disorder, one gets:

$$
\Lambda(g ; \mu ; N)=\frac{1}{N} \overline{\ln \mathcal{N}^{\alpha}\left(\rho^{\alpha} ; \mu ; N\right)}+g \rho(g ; \mu ; N)
$$

where $\rho(g ; \mu ; N)=\overline{\rho^{\alpha}(g ; \mu ; N)}$. A quenched complexity can thus be defined for a finite system as:

$$
\sum_{\mathrm{Q}}^{\mathrm{L}-\mathrm{F}}(\rho ; \mu ; N)=\Lambda(g(\rho ; \mu ; N) ; \mu ; N)-\rho g(\rho ; \mu ; N)
$$

where $g(\rho ; \mu ; N)$ is implicitly defined by $\rho(g ; \mu ; N) . \rho^{\alpha}(g ; \mu ; N)$ is expected to deviate from its average value $\rho$ by terms of order $1 / \sqrt{N}$. As a consequence, $\overline{\ln \mathcal{N}^{\alpha}\left(\rho^{\alpha} ; \mu ; N\right)}$ approaches $\overline{\ln \mathcal{N}^{\alpha}(\rho ; \mu ; N)}$ in the thermodynamic limit, and one has:

$$
\lim _{N \rightarrow \infty} \sum_{\mathrm{Q}}^{\mathrm{L}-\mathrm{F}}(\rho ; \mu ; N)=\sum_{\mathrm{Q}}^{\infty}(\rho ; \mu)
$$

The results for $\Sigma_{\mathrm{Q}}^{\mathrm{L}-\mathrm{F}}(\rho ; \mu ; N)$ are shown in Fig. 10 as a function of $\rho$ and $\mu$, for $N=25$. As previously, the largest values are reached inside the hysteresis loop. But, contrary to previous cases, the quenched complexity exactly vanishes on the main adsorption/desorption hysteresis loop, shown as a solid line in the second panel of Fig. 10. This is expected: it can be seen by inspection of Eq. 7 that the limit $g \rightarrow-\infty$ (respectively $g \rightarrow+\infty$ ) selects the smallest (respectively largest) $\rho$ for which the density of metastable states is not zero, corresponding to the main adsorption (respectively desorption) branches for each realization of disorder. As a consequence, the envelope of the projection in Fig. 10 also corresponds to the average over disorder of the main hysteresis loop. The agreement is quite good within the uncertainties of 
our numerical approach: for each of the $24 \mu$-values used to perform the calculations in the range $[-8.8 ;-8.1], \sum_{\mathrm{Q}}^{\mathrm{L}-\mathrm{F}}(\rho ; \mu ; N=25)=0$ superimpose with the main hysteresis within few percent; the visible discrepancies or roughness in Fig. 10 arise as a consequence of interpolation between the data. In order to discuss the thermodynamic limit, the system size dependence is shown in Fig. 11 for $\mu=-8.55$. It is slightly more pronounced than in previous cases, but still well fitted with the ansatz $\sum+a N^{-1} \ln N+b N^{-1}$, and, as previously, the limiting value of the maximum for $\mu=-8.55$ is compatible with the value previously obtained in section IV for $\sum_{\mathrm{Q}}^{\infty}(\mu=-8.55)$. An extrapolated thermodynamic limit can be proposed (see Fig. 11, dashed line). An interesting feature is that the locations where $\sum_{\mathrm{Q}}^{\mathrm{L}-\mathrm{F}}(\rho ; \mu ; N)=0$ are scarcely dependent on $N$, especially at low adsorption, and thus can be safely extrapolated to large $N$. Therefore, the complexity for $N=25$ shown in Fig. 10 is expected to give a good approximation of the main hysteresis loop in the thermodynamic limit. As mentioned in the Introduction, realistic pores of mesoporous silica are typically few micrometers long, leading to $10^{2}-10^{3}$ domains. The previous argument shows that the complexity shown in Fig. 10 is a fairly good evaluation of the hysteresis for realistic systems.

\section{Systems with macroscopic transitions}

When the disorder is moderate, the hysteresis loop associated to the capillary condensation exhibits an unusual behavior. One observes vertical branches for the main adsorption or desorption curve, generally associated to a true out-of-equilibrium transition characterized by a macroscopic jump in the hysteresis. ${ }^{19}$ Metastable states are expected to be found in the hysteresis loop, but distributed in such a way that some regions are totally depleted..$^{34,36,38}$ 
Such macroscopic transitions are observed in our system, for instance, when the Gaussian distribution for the heterogeneities is replaced by a flat and truncated distribution. ${ }^{33} \mathrm{We}$ suspect that the underlying mechanism is quite different in both situations, for the simple reason that a true out-of-equilibrium transition is not expected in materials exhibiting a onedimensional pore network. The important point is most probably the truncation of the distribution. Gaussian distribution being strictly positive, all heterogeneity values between 0.7 and 1.3 may be found in a given pore. This is not the case if one considers a distribution which is strictly zero for heterogeneity out of a given interval. For instance, we now consider a flat distribution over $[0.9,1.1]$. In this case, the extreme values of the heterogeneity are not observed at all in the pore, while they could be found in our previous example with a Gaussian distribution. From this point of view, the level of disorder is lower.

For any realization of the disorder, a macroscopic transition is observed during adsorption (3 examples are given in Fig. 12 for $N=25$ domains), while desorption occurs in several steps. In all cases, the sharp adsorption is around $\mu=-8.45$, which corresponds to the gas to liquid transition in elementary pores with heterogeneity around its median value. ${ }^{31}$ The small variations around this value yield a rounding of the jump in adsorption after averaging over disorder. However, the larger the system size, the steeper the jump (see Fig. 13). A macroscopic transition is thus suspected in the thermodynamic limit.

All metastable states have been determined and shown in Fig. 12. All of them fall within the main hysteresis loop, but does not distribute uniformly: no metastable state could be found in the right hand part of the hysteresis, except for the gaslike and liquidlike branches. This is explained by noticing that when transition occurs somewhere in the pore for $\mu$ around -8.5 , the meniscus propagates freely in the whole pore (absence of strongly "repulsive" domain to 
stop the meniscus). The system is thus either in the gaslike or the liquidlike state. On the other hand, an exponentially large number of metastable states is found in the left half-region of the hysteresis. Their density is largest around $\rho=0.6$, and decreases monotonously while approaching the gaslike and liquidlike branches.

A quantitative analysis including the average over disorder has been performed by calculating the quenched complexity $\sum_{\mathrm{Q}}^{\mathrm{L}-\mathrm{F}}(\rho ; \mu ; N)$. The result is shown in Fig. 14 for the largest system size $N=30$ and $5 \times 10^{3}$ disorder realizations. A smoothly varying complexity is obtained, with a single maximum for any given chemical potential. As previously noticed by visual inspection on various disorder realizations, the quenched complexity reaches monotonously zero while approaching the main hysteresis loop for $\mu<-8.52$ (Fig. 14, second panel). The interesting behavior is for $\mu>-8.52$ : the quenched complexity is not defined since no metastable states were observed in the 5000 disorder realizations used to perform the average, except for the two gaslike and liquidlike branches. In other words, in this system, the quenched complexity is shown to vanish strictly inside the main hysteresis loop. Before going farther, let us discuss the thermodynamic limit. We have performed the same finite size analysis as in the previous system. The maximum values reached by the quenched complexity slightly decrease while approaching the thermodynamic limit, but, as previously, the region where $\sum_{\mathrm{Q}}^{\mathrm{L}-\mathrm{F}}(\rho ; \mu ; N)=0$ is essentially independent of $N$. In particular, one has $\sum_{\mathrm{Q}}^{\mathrm{L}-\mathrm{F}}(\rho ; \mu ; N)=0$ for $\mu>-8.52$ even in the thermodynamic limit, and thus the quenched complexity vanishes strictly inside the main hysteresis loop.

Such a behavior has been reported in the random field Ising model (RFIM), ${ }^{35,36}$ with however noticeable differences. For instance, in our case, the depleted region extends to all density 
values between the gaslike and the liquidlike states, while, in the RFIM, metastable states could be found in regions close to the main gaslike and/or liquidlike branches (materialized by an ellipse in Fig. 14). The origin for this difference may arise due to the treatment of the interdependence between adjacent domains, or to the particular choice for the fluid/wall interactions and the associated distribution of heterogeneities. It is however suspected that the difference is more fundamental. Remember that the RFIM with the same 1-dimensional topology as our pores predicts a continuous curve whatever the disorder strength. The appearance of a jump in adsorption in our system is closely connected to the truncation of the distribution of heterogeneities. As previously mentioned, the region of chemical potential $\mu>$ -8.52 corresponds to a situation where a liquid/gas meniscus can propagate freely through any heterogeneity in the pore, the truncation of the distribution resulting in the absence of strong enough heterogeneities to pin the meniscus. Furthermore, the gaslike state is metastable while the liquidlike state is stable. As a consequence, any state of intermediate density is unstable and immediately turns into liquidlike state. However, as for the RFIM, the main adsorption loop corresponds to the grand-canonical envelope of the metastable states, where $\sum_{\mathrm{Q}}^{\mathrm{L}-\mathrm{F}}(\rho ; \mu ; N) \geq 0$; it is reminded that along the gaslike and liquidlike branches, the quenched complexity equals zero in the thermodynamic limit.

If the pores are closed at one end, the sharp transition to liquidlike state shifts towards lower value $^{33}(\mu=-8.52)$, which corresponds precisely to the lower limit of the depleted region. This is in agreement with our previous interpretation: the pore end helps to nucleate the liquid/gas meniscus and suppresses the metastable gaslike branch above $\mu=-8.52$. In this situation, the main adsorption/desorption loop is still the envelope of the metastable states, except that one observes a sharp transition during adsorption without depletion of the hysteresis loop (i.e. many intermediate states can be found closely along the sharp transition). This is still different 
from the RFIM results. It is however emphasized that our numerical study cannot exclude a very small depleted region around the transition. Using a finite reservoir may help to investigate these questions. ${ }^{34,39}$

\section{Conclusion}

We have studied numerically the density of metastable states embedded in the main adsorption/desorption hysteresis loop for a simple fluid confined in a single disordered tubular pore. Very few works can be found on such investigations, essentially in lattice gas models. The originality of this study is that the model is based on a molecular simulation approach of the adsorption of a Lennard-Jones fluid in a heterogeneous linear pore. The substrate is divided into domains, and the fluid adsorption properties are pre-determined for each domain by molecular simulation as a function of the fluid state in the neighboring domains, thus explicitly taking into account the effect of interconnections between domains. This approach completely differs from pore network models where ad-hoc rules for filling or emptying are postulated (pore blocking, etc.). The adsorption properties of the entire pore can be then calculated straightforwardly in our approach, which allows to characterize all the numerous metastable states. The annealed and quenched averages over the disorder originating in the physico-chemical heterogeneities in the pore are introduced to calculate the corresponding complexities. Finite size effects have been studied extensively. Interestingly, they are shown to be quite small concerning the total number of metastable state at a given chemical potential, and the data can be safely extrapolated to the thermodynamic limit. The annealed and quenched complexities, characterizing the density of metastable states, present more pronounced finite size effects; the thermodynamic limit is inferred from the expected $1 / N$ and 
$\ln N / N$ behavior. When possible, the numerical results have been compared to exact analytical calculations, validating the average over disorder and the finite size analysis.

One observes that the density of metastable states grows exponentially with system size and presents a pronounced maximum in the central region of the hysteresis. The annealed complexity remains positive in a region which extends beyond the main average hysteresis loop, confirming that the average density of metastable states is unsuited to characterize the hysteresis in the thermodynamic limit. On the other hand, the quenched complexity, characterizing the typical number of metastable states, is shown to be strictly positive only inside the hysteresis and to vanish on the main grand canonical adsorption and desorption branches. Equivalently, the main adsorption/desorption hysteresis averaged over disorder corresponds to the envelope of the typical distribution of metastable states. An interesting point concerns the comparison of two systems with different distribution of heterogeneities, one Gaussian, the other one flat and truncated. The main difference is that in the second one the high values of disorder are strictly suppressed. In the first case, a smooth hysteresis is observed, and an exponentially large density of metastable states is found everywhere in the loop. In the second case, a macroscopic jump is observed during adsorption, and a depleted region with no metastable states appears in the vicinity of the transition within the hysteresis loop. The interpretation is as follows: The distribution of heterogeneities being truncated, the less attractive domains are not strong enough to impede the propagation of a meniscus. As a consequence, the possible states are just liquidlike and gaslike. The first one is actually the stable state. The gaslike state is metastable due to the truncation of the distribution: the most attractive regions in the pore are not strong enough to nucleate a liquid meniscus. When the pores are closed at one end, the depleted region and the hysteresis shrink, as a consequence of the facile nucleation of a meniscus in the bottom, while the transition remains sharp and 
macroscopic. Further investigation is needed to assess whether the depleted region completely disappears or remains as a thin area close to the transition. Significantly larger systems are required to minimize finite size effects and reach the required resolution for the quenched complexity.

Acknowledgement: Dr. E. Kierlik and Dr. G. Tarjus are gratefully acknowledged for helpful discussions. The simulations were performed thanks to grant from the Institut de Développement des Ressources en Informatique Scientifique IDRIS-CNRS, Orsay, France. 


\section{Appendices:}

These appendices present the main steps in deriving the analytic formulae for the annealed and quenched complexities when the domains can be separated in two types (the so-called Atype and B-type, see below); i.e. for $\mu<-8.53$.

\section{A: Annealed and quenched complexities for finite size system}

Examination of the various possible conditional isotherms induced by disorder shows that for $\mu<-8.53$, all domains necessarily fall into one of two types (see Ref $\left[{ }^{31}\right]$ and Fig. 1). Type A corresponds to domains for which $\mu_{\text {coex }}<\mu<\mu_{2}$ occurring when the disorder parameter $h>$ 0.945 ( $h$ is the modulation factor of the fluid/wall interaction). ${ }^{31}$ Such domains can be in two states (G or L) only if both neighbors are gaslike. Otherwise, these domains are necessarily in the liquidlike state. Type B corresponds to domains for which $\mu_{1}<\mu<\mu_{\text {coex }}$, occurring for $h$ $<0.945$. They can be in two states ( $\mathrm{G}$ or $\mathrm{L})$ only if both neighbors are liquidlike. Otherwise, these domains are necessarily in the gaslike state. For our particular choice of $\mu$, any pore can thus be seen as a chain of A's and B's, and the total number of domains is $N=N_{\mathrm{A}}+N_{\mathrm{B}}$ where $N_{\mathrm{A}}$ and $N_{\mathrm{B}}$ denote the number of domains $\mathrm{A}$ and $\mathrm{B}$. Knowing the relation between the domain type and $h$ as well as the distribution of $h$, one can deduce that the probability of having a domain $\mathrm{A}$ is $p_{\mathrm{A}}=0.625$ (and $p_{\mathrm{B}}=0.375$ ). Considering the properties of domains $\mathrm{A}$ and $\mathrm{B}$, if two A's or two B's are in contact they are necessarily in the same fluid state. This can be generalized to any group of A's or B's. The pore can thus be seen as a chain of A- and Bregions of various lengths. $N_{\mathrm{A}}^{\mathrm{R}}$ and $N_{\mathrm{B}}^{\mathrm{R}}$ denote the number of A-regions and B-regions, and 
$N^{\mathrm{R}}=N_{\mathrm{A}}^{\mathrm{R}}+N_{\mathrm{B}}^{\mathrm{R}}$ the total number of regions. Any metastable fluid state in the pore can be described by a chain of $N^{\mathrm{R}}$ fluid states corresponding to the $N^{\mathrm{R}}$ regions of the pore. It is also noted that any B-region at one pore end is necessarily in the gaslike state. $N_{\mathrm{B}}^{\mathrm{e}}$ denotes the number of domains B belonging to B-regions which are at the pore ends. If the pore has an Adomain at both ends, then $N_{\mathrm{B}}^{\mathrm{e}}=0$. We first calculate the possible values for the number $\mathcal{N}(\mu=-8.55 ; N)$ of metastable states (for various disorder realizations). The $N_{\mathrm{B}}^{\mathrm{e}}$ B-domains do not contribute to $\mathcal{N}(\mu=-8.55 ; N)$. The relevant quantity is $N_{\mathrm{A}}^{\mathrm{R}}$. We denote $\mathcal{N}\left(N_{\mathrm{A}}^{\mathrm{R}}\right)$ the number of metastable states for a chain of $N_{\mathrm{A}}^{\mathrm{R}}$ A-regions (the number of B-regions is necessarily $N_{\mathrm{A}}^{\mathrm{R}}-1, N_{\mathrm{A}}^{\mathrm{R}}$, or $N_{\mathrm{A}}^{\mathrm{R}}+1$ depending of the nature of pore ends. Simple inspection shows that $\mathcal{N}\left(N_{\mathrm{A}}^{\mathrm{R}}=0\right)=1, \mathcal{N}\left(N_{\mathrm{A}}^{\mathrm{R}}=1\right)=2$. Let us consider the last A-region of the pore (for $N_{\mathrm{A}}^{\mathrm{R}} \geq 1$ ). The fluid state in this region is either G or L. We denote $\mathcal{N}^{\mathrm{G}}\left(N_{\mathrm{A}}^{\mathrm{R}}\right)$ the number of metastable states which are gaslike in this region, and $\mathcal{N}^{\mathrm{L}}\left(N_{\mathrm{A}}^{\mathrm{R}}\right)$ the number of metastable states which are liquidlike in this region. One has the following relations:

$$
\begin{aligned}
& \mathcal{N}\left(N_{\mathrm{A}}^{\mathrm{R}}\right)=\mathcal{N}^{\mathrm{G}}\left(N_{\mathrm{A}}^{\mathrm{R}}\right)+\mathcal{N}^{\mathrm{L}}\left(N_{\mathrm{A}}^{\mathrm{R}}\right) \\
& \mathcal{N}^{\mathrm{G}}\left(N_{\mathrm{A}}^{\mathrm{R}}+1\right)=\mathcal{N}^{\mathrm{G}}\left(N_{\mathrm{A}}^{\mathrm{R}}\right)+\mathcal{N}^{\mathrm{L}}\left(N_{\mathrm{A}}^{\mathrm{R}}\right) \\
& \mathcal{N}^{\mathrm{L}}\left(N_{\mathrm{A}}^{\mathrm{R}}+1\right)=\mathcal{N}^{\mathrm{G}}\left(N_{\mathrm{A}}^{\mathrm{R}}\right)+2 \mathcal{N}^{\mathrm{L}}\left(N_{\mathrm{A}}^{\mathrm{R}}\right) \\
& \text { and } \mathcal{N}^{\mathrm{G}}\left(N_{\mathrm{A}}^{\mathrm{R}}=1\right)=\mathcal{N}^{\mathrm{L}}\left(N_{\mathrm{A}}^{\mathrm{R}}=1\right)=1 .
\end{aligned}
$$

Straightforward calculations show that $\mathcal{N}\left(N_{\mathrm{A}}^{\mathrm{R}}\right)$ successively takes the values $1,2,5,13 \ldots$ or:

$$
\mathcal{N}\left(N_{\mathrm{A}}^{\mathrm{R}}\right)=\alpha_{1} \lambda_{1}^{N_{\mathrm{R}}^{\mathrm{R}}}+\alpha_{2} \lambda_{2}^{N_{\mathrm{A}}^{\mathrm{R}}}
$$


with $\alpha_{1}=(5+\sqrt{5}) / 10, \alpha_{2}=(5-\sqrt{5}) / 10, \lambda_{1}=(3+\sqrt{5}) / 2$, and $\lambda_{2}=(3-\sqrt{5}) / 2$. This gives the possible values taken by $\mathcal{N}(\mu=-8.55 ; N)$ depending on the disorder configuration. We now calculate their probability.

For $N$ domains, the probability to have $N_{\mathrm{A}}^{\mathrm{R}}$ A-regions is given by:

$\mathcal{P}\left(N_{\mathrm{A}}^{\mathrm{R}} ; N\right)=\sum_{N_{\mathrm{B}}^{\mathrm{e}}=0}^{N-2 N_{\mathrm{A}}^{\mathrm{R}}+1} \sum_{N_{\mathrm{A}}=N_{\mathrm{A}}^{\mathrm{R}}}^{N-N_{\mathrm{B}}^{\mathrm{e}}-N_{\mathrm{A}}^{\mathrm{R}}+1}\left(\begin{array}{l}N_{\mathrm{A}}-1 \\ N_{\mathrm{A}}^{\mathrm{R}}-1\end{array}\right)\left(\begin{array}{l}N-N_{\mathrm{A}}-N_{\mathrm{B}}^{\mathrm{e}}-1 \\ N_{\mathrm{A}}^{\mathrm{R}}-2\end{array}\right)\left(N_{\mathrm{B}}^{\mathrm{e}}+1\right) p_{\mathrm{A}}^{N_{\mathrm{A}}} p_{\mathrm{B}}^{N-N_{\mathrm{A}}}$

where $\left(\begin{array}{l}N_{\mathrm{A}}-1 \\ N_{\mathrm{A}}^{\mathrm{R}}-1\end{array}\right)$ is the number of ways to split the $N_{\mathrm{A}}$ domains into $N_{\mathrm{A}}^{\mathrm{R}}$ A-regions, and $\left(\begin{array}{c}N-N_{\mathrm{A}}-N_{\mathrm{B}}^{\mathrm{e}}-1 \\ N_{\mathrm{A}}^{\mathrm{R}}-2\end{array}\right)$ is the number of ways to split the $N-N_{\mathrm{A}}-N_{\mathrm{B}}^{\mathrm{e}}$ B-domains which are not at pore ends into $N_{\mathrm{A}}^{\mathrm{R}}-1$ B-regions which are in-between the $N_{\mathrm{A}}^{\mathrm{R}}$ A-regions. The parenthesis $\left(N_{\mathrm{B}}^{\mathrm{e}}+1\right)$ takes into account the number of ways to append the $N_{\mathrm{B}}^{\mathrm{e}}$ B-domains at both pore ends. The summations are performed over all possible choices for $N_{\mathrm{B}}^{\mathrm{e}}$ and $N_{\mathrm{A}}$ compatible with $N_{\mathrm{A}}^{\mathrm{R}}$ Aregions. The probabilities $\mathscr{P}\left(N_{\mathrm{A}}^{\mathrm{R}} ; N\right)$ can easily be obtained numerically, which allows to calculate the quenched and annealed complexities as a function of $N$ for $\mu=-8.55$. The results are shown as red solid lines in Fig. 4.

\section{B: Annealed and quenched complexities in the thermodynamic limit}

The thermodynamic limit of the quenched and annealed complexities (Eqs. 3 and 4) involve the $N \rightarrow \infty$ limit. We thus calculate an approximate formula for $\mathscr{P}\left(N_{\mathrm{A}}^{\mathrm{R}} ; N\right)$ for large $N$ (Eq. A5). Let us denote $N_{\mathrm{A}}^{\mathrm{R} \text {;max }}$ the largest possible value for the number of A-regions (see Appendix A). $N_{\mathrm{A}}^{\mathrm{R} \text {;max }}$ is $N / 2$ if $N$ is even, and $(N+1) / 2$ if $N$ is odd. For large $N$ one has 
$N_{\mathrm{A}}^{\mathrm{R} ; \max } \approx N / 2$. Inspection of Eq. A5 shows that $\mathcal{P}\left(N_{\mathrm{A}}^{\mathrm{R}} ; N\right)$ has negligible values if $N_{\mathrm{A}} \ll N_{\mathrm{A}}^{\mathrm{R} ; \max }$. We introduce the reduced quantities $n_{\mathrm{A}}^{\mathrm{R}}=N_{\mathrm{A}}^{\mathrm{R}} / N, n_{\mathrm{B}}^{\mathrm{e}}=N_{\mathrm{B}}^{\mathrm{e}} / N$, and $n_{\mathrm{A}}=N_{\mathrm{A}} / N$. One has:

$0 \leq n_{\mathrm{A}}^{\mathrm{R}} \leq 0.5,0 \leq n_{\mathrm{B}}^{\mathrm{e}} \leq 1-2 n_{\mathrm{A}}^{\mathrm{R}}+1 / N$, and $n_{\mathrm{A}}^{\mathrm{R}} \leq n_{\mathrm{A}} \leq 1-n_{\mathrm{B}}^{\mathrm{e}}-n_{\mathrm{A}}^{\mathrm{R}}+1 / N$

Since now on, all quantities negligible in the thermodynamic limit are dropped. Using Stirling formula:

$\left(\begin{array}{c}M \\ x M\end{array}\right) \underset{M \rightarrow \infty}{\approx} e^{-M[x \ln x+(1-x) \ln (1-x)]+\mathrm{o}(M)}$

and replacing the discrete sums by integrals, the probability $\mathcal{P}\left(N_{\mathrm{A}}^{\mathrm{R}} ; N\right)$ can be written as:

$$
\mathcal{P}\left(N_{\mathrm{A}}^{\mathrm{R}}=n_{\mathrm{A}}^{\mathrm{R}} N ; N\right) \underset{N \rightarrow \infty}{\approx} \iint_{\mathcal{D}} n_{\mathrm{B}}^{\mathrm{e}} e^{N \phi\left(p_{A}, p_{B}, n_{\mathrm{A}}^{\mathrm{R}}, n_{\mathrm{B}}^{\mathrm{e}}, n_{\mathrm{A}}\right)+\mathrm{o}(N)} d n_{\mathrm{B}}^{\mathrm{e}} d n_{\mathrm{A}}
$$

where the integral is performed over the domain $\mathscr{D}$ defined by Eq. B1 (the terms $1 / N$ are neglected) and

$$
\begin{aligned}
& \phi\left(p_{A}, p_{B}, n_{\mathrm{A}}^{\mathrm{R}}, n_{\mathrm{B}}^{\mathrm{e}}, n_{\mathrm{A}}\right)=n_{\mathrm{A}} \ln p_{A}+\left(1-n_{\mathrm{A}}\right) \ln p_{B}+n_{\mathrm{A}} \ln n_{A}+\left(1-n_{\mathrm{A}}-n_{\mathrm{B}}^{\mathrm{e}}\right) \ln \left(1-n_{\mathrm{A}}-n_{\mathrm{B}}^{\mathrm{e}}\right) \\
&-\left(n_{\mathrm{A}}-n_{\mathrm{A}}^{\mathrm{R}}\right) \ln \left(n_{\mathrm{A}}-n_{\mathrm{A}}^{\mathrm{R}}\right)-\left(1-n_{\mathrm{A}}-n_{\mathrm{B}}^{\mathrm{e}}-n_{\mathrm{A}}^{\mathrm{R}}\right) \ln \left(1-n_{\mathrm{A}}-n_{\mathrm{B}}^{\mathrm{e}}-n_{\mathrm{A}}^{\mathrm{R}}\right)-2 n_{\mathrm{A}}^{\mathrm{R}} \ln n_{\mathrm{A}}^{\mathrm{R}} .
\end{aligned}
$$

The dominant term of the integral is given by the maximum of $\phi$ over $\mathcal{D} . \phi$ is strictly decreasing with $n_{\mathrm{B}}^{\mathrm{e}}$ and thus the maximum is for $n_{\mathrm{B}}^{\mathrm{e}}=0$, i.e. when the pore has A-domains at both ends. Let us now consider $\phi\left(p_{A}, p_{B}, n_{\mathrm{A}}^{\mathrm{R}}, n_{\mathrm{B}}^{\mathrm{e}}=0, n_{\mathrm{A}}\right)$ as a function of $n_{\mathrm{A}}$. For $n_{\mathrm{A}}^{\mathrm{R}} \neq 0.5$ this function presents a maximum for $n_{\mathrm{A}}^{*}$ verifying:

$$
\frac{\left(n_{\mathrm{A}}^{*}-n_{\mathrm{A}}^{\mathrm{R}}\right)\left(1-n_{\mathrm{A}}^{*}\right)}{n_{\mathrm{A}}^{*}\left(1-n_{\mathrm{A}}^{*}-n_{\mathrm{A}}^{\mathrm{R}}\right)}=\frac{p_{\mathrm{A}}}{p_{\mathrm{B}}}
$$

For a given $n_{\mathrm{A}}^{\mathrm{R}}$, the 1.h.s is an increasing function from 0 to infinity for $n_{\mathrm{A}}^{*}$ running from $n_{\mathrm{A}}^{\mathrm{R}}$ to $1-n_{\mathrm{A}}^{\mathrm{R}}$. The solution thus exists and one has $n_{\mathrm{A}}^{\mathrm{R}}<n_{\mathrm{A}}^{*}<1-n_{\mathrm{A}}^{\mathrm{R}}$. Furthermore, the 1.h.s. is 
always equal to 1 for $n_{\mathrm{A}}^{*}=0.5$ As a consequence, for $p_{\mathrm{A}}>p_{\mathrm{B}}$ (respectively $p_{\mathrm{A}}<p_{\mathrm{B}}$ ) one has $n_{\mathrm{A}}^{*}>0.5$ (respectively $n_{\mathrm{A}}^{*}<0.5$ ). The maximum of $\phi$ is given by:

$\phi^{\max }\left(p_{A}, p_{B}, n_{\mathrm{A}}^{\mathrm{R}}\right)=\phi\left(p_{A}, p_{B}, n_{\mathrm{A}}^{\mathrm{R}}, n_{\mathrm{B}}^{\mathrm{e}}=0, n_{\mathrm{A}}=n_{\mathrm{A}}^{*}\right)$

For $n_{\mathrm{A}}^{\mathrm{R}}=0.5, n_{\mathrm{A}}$ can only take the value 0.5 , thus $n_{\mathrm{A}}^{*}=0.5$ and the previous equation still holds.

The probability $\mathscr{P}\left(N_{\mathrm{A}}^{\mathrm{R}} ; N\right)$ is thus:

$\mathscr{P}\left(N_{\mathrm{A}}^{\mathrm{R}}=n_{\mathrm{A}}^{\mathrm{R}} N ; N\right) \underset{N \rightarrow \infty}{\approx} e^{N \phi^{\max }\left(p_{A}, p_{B}, n_{\mathrm{A}}^{\mathrm{R}}\right)+\mathrm{O}(N)}$

$\phi^{\max }\left(p_{A}, p_{B}, n_{\mathrm{A}}^{\mathrm{R}}\right)$, seen as a function of $n_{\mathrm{A}}^{\mathrm{R}}$ exhibits a maximum for $n_{\mathrm{A}}^{\mathrm{R}^{*}}$ (the corresponding value of $n_{\mathrm{A}}^{*}$ is denoted $n_{\mathrm{A}}^{* *}$ ) such that

$\left.\frac{d \phi^{\max }\left(p_{A}, p_{B}, n_{\mathrm{A}}^{\mathrm{R}}\right)}{d n_{\mathrm{A}}^{\mathrm{R}}}\right|_{n_{\mathrm{A}}^{\mathrm{R}}=n_{\mathrm{A}}^{\mathrm{R}^{*}}}=\left.\frac{\partial \phi\left(p_{A}, p_{B}, n_{\mathrm{A}}^{\mathrm{R}}, n_{\mathrm{B}}^{\mathrm{e}}=0, n_{\mathrm{A}}\right)}{\partial n_{\mathrm{A}}^{\mathrm{R}}}\right|_{n_{\mathrm{A}}^{\mathrm{R}}=n_{\mathrm{A}}^{\mathrm{R}^{*}} ; n_{\mathrm{A}}=n_{\mathrm{A}}^{* *}}=0$

The first equality holds since by definition $\left.\frac{\partial \phi\left(p_{A}, p_{B}, n_{\mathrm{A}}^{\mathrm{R}}, n_{\mathrm{B}}^{\mathrm{e}}=0, n_{\mathrm{A}}\right)}{\partial n_{\mathrm{A}}}\right|_{n_{\mathrm{A}}=n_{\mathrm{A}}^{* * *}}=0$. The result is $\left(1-n_{\mathrm{A}}^{* *}-n_{\mathrm{A}}^{\mathrm{R}^{*}}\right)\left(n_{\mathrm{A}}^{* *}-n_{\mathrm{A}}^{\mathrm{R}^{*}}\right)=\left(n_{\mathrm{A}}^{\mathrm{R} *}\right)^{2}$, which gives, using Eq. B5: $n_{\mathrm{A}}^{\mathrm{R}^{*}}=p_{\mathrm{A}} p_{\mathrm{B}}$ and $n_{\mathrm{A}}^{* *}=p_{A}$. Inserting these values into Eq. B6 shows that this maximum is actually zero. Hence, $\phi^{\max }\left(p_{A}, p_{B}, n_{\mathrm{A}}^{\mathrm{R}}\right) \leq 0$ and the leading term of the probability given by Eq. B7 is correctly normalized. Note that the probability $\mathscr{P}\left(N_{\mathrm{A}}^{\mathrm{R}} ; N\right)$ is thus essentially Gaussian around $n_{\mathrm{A}}^{\mathrm{R} *}=p_{\mathrm{A}} p_{\mathrm{B}}:$

$\mathscr{P}\left(N_{\mathrm{A}}^{\mathrm{R}}=n_{\mathrm{A}}^{\mathrm{R}} N ; N\right) \underset{N \rightarrow \infty}{\approx} e^{-N \frac{\lambda}{2}\left(n_{\mathrm{A}}^{\mathrm{R}}-p_{\mathrm{A}} p_{\mathrm{B}}\right)^{2}+\mathrm{O}(N)}$

with $\lambda=-\partial^{2} \phi^{\max }\left(p_{A}, p_{B}, n_{\mathrm{A}}^{\mathrm{R}}\right) /\left.\partial n_{\mathrm{A}}^{\mathrm{R}^{2}}\right|_{n_{\mathrm{A}}^{\mathrm{R}}=n_{\mathrm{A}}^{\mathrm{R}^{*}}}=\left(p_{\mathrm{A}} p_{\mathrm{B}}\right)^{-2}>0$. 


\section{Annealed complexity:}

The annealed complexity is given by

$$
\sum_{\mathrm{A}}^{\infty}(\rho ; \mu)=\lim _{N \rightarrow \infty} \frac{1}{N} \ln \overline{\mathcal{N}(\rho ; \mu ; N)}=\lim _{N \rightarrow \infty} \frac{1}{N} \ln \left[\sum_{q_{\mathrm{A}}=0}^{N_{\mathrm{A}}^{R ; n a x}} \mathcal{P}\left(N_{\mathrm{A}}^{\mathrm{R}} ; N\right) \mathcal{N}\left(N_{\mathrm{A}}^{\mathrm{R}}\right)\right]
$$

Turning the discrete sum into integral gives:

$$
\sum_{q_{\mathrm{A}}=0}^{N_{\mathrm{A}}^{R ; m a x}} \mathcal{P}\left(N_{\mathrm{A}}^{\mathrm{R}} ; N\right) \mathcal{N}\left(N_{\mathrm{A}}^{\mathrm{R}}\right)=\alpha \int_{-\infty}^{+\infty} N\left[e^{\max }\left(p_{A}, p_{B}, n_{\mathrm{A}}^{\mathrm{R}}\right)+n_{\mathrm{A}}^{\mathrm{R}} \ln \lambda_{1}\right]+\mathrm{o}(N) d n_{\mathrm{A}}^{\mathrm{R}}
$$

The integral of the exponential is again dominated by its largest term, obtained here for $n_{\mathrm{A}}^{\mathrm{R}} \equiv n_{\mathrm{A}}^{\mathrm{R} ; \mathrm{Q}}$ (the corresponding value of $n_{\mathrm{A}}^{*}$ is denoted $n_{\mathrm{A}}^{* ; \mathrm{Q}}$ ) such that:

$$
\begin{gathered}
\left.\frac{d \phi^{\max }\left(p_{A}, p_{B}, n_{\mathrm{A}}^{\mathrm{R}}\right)}{d n_{\mathrm{A}}^{\mathrm{R}}}\right|_{n_{\mathrm{A}}^{\mathrm{R}}=n_{\mathrm{A}}^{\mathrm{R}: \mathrm{Q}}}=\left.\frac{\partial \phi^{\max }\left(p_{A}, p_{B}, n_{\mathrm{A}}^{\mathrm{R}}\right)}{\partial n_{\mathrm{A}}^{\mathrm{R}}}\right|_{n_{\mathrm{A}}^{\mathrm{R}}=n_{\mathrm{A}}^{\mathrm{R}: \mathrm{Q}}}=-\ln \lambda_{1} \\
\Leftrightarrow \ln \left[\lambda_{1} \frac{\left(1-n_{\mathrm{A}}^{* ; \mathrm{Q}}-n_{\mathrm{A}}^{\mathrm{R} ; \mathrm{Q}}\right)\left(n_{\mathrm{A}}^{* ; \mathrm{Q}}-n_{\mathrm{A}}^{\mathrm{R} ; \mathrm{Q}}\right)}{\left(n_{\mathrm{A}}^{\mathrm{R} ; \mathrm{Q}}\right)^{2}}\right]=0
\end{gathered}
$$

The first equality holds for by definition $\left.\frac{\partial \phi\left(p_{A}, p_{B}, n_{\mathrm{A}}^{\mathrm{R}}, n_{\mathrm{B}}^{\mathrm{e}}=0, n_{\mathrm{A}}\right)}{\partial n_{\mathrm{A}}}\right|_{n_{\mathrm{A}}=n_{\mathrm{A}}^{*} \mathrm{Q}}=0$. The results are:

$$
\begin{aligned}
& n_{\mathrm{A}}^{\mathrm{R} ; \mathrm{Q}}=\left(\frac{1+\sqrt{5}}{4}\right)\left(1-\frac{1}{\sqrt{1+2(1+\sqrt{5}) p_{\mathrm{A}} p_{\mathrm{B}}}}\right) \\
& n_{\mathrm{A}}^{* ; \mathrm{Q}}=\frac{1}{2}\left(1+\operatorname{sgn}\left(p_{\mathrm{A}}-p_{\mathrm{B}}\right) \sqrt{\frac{1-4 p_{\mathrm{A}} p_{\mathrm{B}}}{1+2(1+\sqrt{5}) p_{\mathrm{A}} p_{\mathrm{B}}}}\right)
\end{aligned}
$$

Where $\operatorname{sgn}\left(p_{\mathrm{A}}-p_{\mathrm{B}}\right)$ is the sign of $p_{\mathrm{A}}-p_{\mathrm{B}}$. For $p_{\mathrm{A}}=0.625$ we find numerically $n_{\mathrm{A}}^{\mathrm{R} ; \mathrm{Q}}=0.2990711$ and $n_{\mathrm{A}}^{* ; \mathrm{Q}}=0.5787910$, and using Eqs. $\mathrm{B} 4$ and $\mathrm{B} 6$, one gets $\sum_{\mathrm{A}}^{\infty}(\rho ; \mu=-8.55)=0.25715$ 


\section{Quenched complexity:}

The quenched complexity is given by:

$\sum_{\mathrm{Q}}^{\infty}(\rho ; \mu)=\lim _{N \rightarrow \infty} \frac{1}{N} \overline{\ln \mathcal{N}(\rho ; \mu ; N)}=\lim _{N \rightarrow \infty} \frac{1}{N} \sum_{q_{\mathrm{A}}=0}^{N_{\mathrm{A}}^{R} \max } \mathcal{P}\left(N_{\mathrm{A}}^{\mathrm{R}} ; N\right) \ln \mathcal{N}\left(N_{\mathrm{A}}^{\mathrm{R}}\right)$

Using Eqs. A4 and B7 and the fact that the integral of the exponential is dominated by its largest term in the thermodynamic limit (obtained for $n_{\mathrm{A}}^{\mathrm{R}} \equiv n_{\mathrm{A}}^{\mathrm{R} ; \mathrm{A}}=n_{\mathrm{A}}^{\mathrm{R} *}$ ), one gets.

$\sum_{\mathrm{Q}}^{\infty}(\rho ; \mu)=\ln \lambda_{1} \lim _{N \rightarrow \infty} \frac{1}{N} \sum_{q_{\mathrm{A}}=0}^{N_{\mathrm{A}}^{R \text { max }}} N_{\mathrm{A}}^{\mathrm{R}} \mathcal{P}\left(N_{\mathrm{A}}^{\mathrm{R}} ; N\right)=p_{\mathrm{A}} p_{\mathrm{B}} \ln \lambda_{1}$ 


\section{References}

J. S. Beck, J. C. Vartuli, W. J. Roth, M. E. Leonowicz, C. T. Kresge, K. D. Schmitt, C. T.-W. Chu, D. H. Olson, E. W. Sheppard, S. B. McCullen, J. B. Higgins, and J. L. Schlenker, J. Am. Chem. Soc. 114, 10834 (1992).

C. T. Kresge, M. E. Leonowicz, W. J. Roth, J. C. Vartuli, and J. S. Beck, Nature 359, 710 (1992).

A. Uhlir, Bell Syst. Tech. 35, 333 (1956).

4 (2002).

D. Wallacher, N. Künzner, D. Kovalev, N. Knorr, and K. Knorr, Phys. Rev. Lett. 92 (19), 195704 (2004).

K. Morishige and M. Ishino, Langmuir 23 (22), 11021 (2007).

L. D. Gelb, K. E. Gubbins, R. Radhakrishnan, and M. Sliwinska-Bartkowiak, Rep. Prog. Phys. 62, 1573 (1999).

M. D. Tascon, and A. Linares-Solano, in Studies in Surface Science and Catalysis, edited by F. Rodriguez Reinoso, B. McEnaney, J. Rouquerol, and K. Unger (Elsevier Science, Amsterdam, 2002), Vol. 144, pp. 83. 
V. B. Fenelonov, A. Y. Derevyankin, S. D. Kirik, L. A. Solovyov, A. N. Shmakov, J.L. Bonardet, A. Gedeon, and V. N. Romannikov, Micropor. Mesopor. Mater. 44-45, $33(2001)$.

C. G. Sonwane, C. W. Jones, and P. J. Ludovice, J. Phys. Chem. B 109 (49), 23395 (2005).

R. Guégan, D. Morineau, C. Loverdo, W. Béziel, and M. Guendouz, Phys. Rev. E 73 (1), 011707 (2006).

B. Coasne, F. R. Hung, R. J.-M. Pellenq, F. R. Siperstein, and K. E. Gubbins, Langmuir 22 (1), 194 (2006).

E. Kierlik, M. L. Rosinberg, G. Tarjus, and P. Viot, Phys. Chem. Chem. Phys. 3 (7), $1201(2001)$.

E. Kierlik, P. A. Monson, M. L. Rosinberg, L. Sarkisov, and G. Tarjus, Phys. Rev. Lett. 87 (5), 055701 (2001).

E. Kierlik, P. A. Monson, M. L. Rosinberg, and G. Tarjus, J. Phys.: Condens. Matter 14, 9295 (2002). 
G. Mason, J. Colloid Interface Sci. 88 (1), 36 (1982).

J. Puibasset, J. Chem. Phys. 127 (15), 154701 (2007).

S. Naumov, A. Khokhlov, R. Valiullin, J. Kärger, and P. A. Monson, Phys. Rev. E 78 (6), 060601 (2008).

J. Puibasset, Langmuir 25 (2), 903 (2009).

E. Kierlik, J. Puibasset, and G. Tarjus, J. Phys.: Condens. Matter 21 (15), 155102 (2009). (2008). 


\section{Figure captions:}

Figure 1: Schematic representation of the typical set of 4 "conditional" isotherms characterizing the fluid adsorption properties in a single cylindrical domain, depending on the fluid state in the two neighboring domains: $\mathrm{G}$ is for gaslike, and $\mathrm{L}$ is for liquidlike. Due to symmetry, the (reversible) G-L and L-G isotherms are identical. Note the hysteresis in the two other situations, and the arrows on the irreversible vertical branches. The isotherms are slightly shifted for clarity.

Figure 2: Black curves: main adsorption/desorption hysteresis. Red lines: all metastable states obtained by systematic exploration. The total number of metastable states $\mathcal{N}$ is given in each figure. From left to right: increasing number of domains $(N=5,10$, and 15). For each $N$, 3 disorder realizations are shown. Insert: enlargement of the high metastable state density region.

Figure 3: Upper panels: Distribution of the number of states $\mathcal{N}$ found in pores made of $N=$ 10 and 20 domains. The distributions cannot be displayed on a single figure due to scale. Lower panel: circles: distributions of the natural logarithm of $\mathcal{N}$, for various $N$; the solid lines are Gaussian fits. All distributions were obtained for $5 \times 10^{3}$ disorder realizations. Insert: variance of the Gaussian fits versus $N$.

Figure 4: System size dependence of the annealed and quenched complexities for $\mu=-8.55$. Circles: simulation data. The dashed lines are fits to the data, and the solid curves are the theoretical results (see text and Appendix A). The horizontal straight lines give the theoretical thermodynamic limits (see Appendix B). 
Figure 5: Quenched and annealed complexities (Eq. 3 and 4) versus chemical potential. Symbols: exact enumeration. Circles: $N=15\left(10^{5}\right.$ disorder realizations); squares: $N=20\left(10^{5}\right.$ disorder realizations); up triangles: $N=25\left(5 \times 10^{4}\right.$ disorder realizations); down triangles: $N=$ $30\left(5 \times 10^{3}\right.$ disorder realizations). Solid lines: theoretical thermodynamic limit (see text and Appendix B) for $\mu<-8.53$.

Figure 6: Annealed complexity $\Sigma_{\mathrm{A}}(\rho ; \mu, N)$ versus $\mu$ and $\rho$ for $N=25$. The same colors are used in both representations. The solid line in the right panel is the average main hysteresis loop for $N=25$. The average is performed for $5 \times 10^{4}$ disorder realizations.

Figure 7: Symbols: annealed complexity versus amount adsorbed $\rho$ for $N=15,20,25,30$ and $\mu=-8.55$ (lines are guides to the eye). Number of disorder realizations for $N=15,20,25$ and 30: $10^{5}, 10^{5}, 5 \times 10^{4}$, and $5 \times 10^{3}$ respectively. Dashed line: extrapolated thermodynamic limit (see text).

Figure 8: Quenched complexity $\sum_{\mathrm{Q}}^{\mathrm{Ch}}(\rho ; \mu ; N)=N^{-1} \overline{\ln \mathcal{N}(\rho ; \mu ; N)}$ versus $\mu$ and $\rho$ for $N=25$, where the average is performed with the prescription that $\ln 0=0$ (see text). The same colors are used in both representations. The solid line in the right panel is the average main hysteresis loop for $N=25$. The average is performed for $5 \times 10^{4}$ disorder realizations.

Figure 9: Quenched complexity $\sum_{\mathrm{Q}}^{\mathrm{Ch}}(\rho ; \mu ; N)=N^{-1} \overline{\ln \mathcal{N}(\rho ; \mu ; N)}$ versus $\rho$ for $\mu=-8.55$ and $N=15,20,25$, and 30 . The average is performed with the prescription that $\ln 0=0$ (see text). Number of disorder realizations for $N=15,20,25$ and $30: 10^{5}, 10^{5}, 5 \times 10^{4}$, and $5 \times 10^{3}$ respectively. Dashed line: extrapolated thermodynamic limit (see text). 
Figure 10: Quenched complexity $\sum_{\mathrm{Q}}^{\mathrm{L}-\mathrm{F}}(\rho ; \mu ; N)$ versus $\mu$ and $\rho$ for $N=25$. The colors are identical in both representations. The solid line in the right panel is the average main hysteresis loop for $N=25$. The average is performed for $5 \times 10^{4}$ disorder realizations.

Figure 11: Quenched complexity $\sum_{\mathrm{Q}}^{\mathrm{L}-\mathrm{F}}(\rho ; \mu ; N)$ versus $\rho$ for $N=15,20,25,30$ and $\mu=-8.55$. Number of disorder realizations for $N=15,20,25$ and $30: 10^{5}, 10^{5}, 5 \times 10^{4}$, and $5 \times 10^{3}$ respectively. The dashed line is the extrapolated thermodynamic limit (see text).

Figure 12: Main adsorption/desorption hysteresis (black curves) and all metastable states (red lines) obtained by systematic exploration. The total number of metastable states $\mathcal{N}$ is given in each panel, corresponding to three realizations of the disorder. The number of domains is $N$ $=25$.

Figure 13: Enlargement of the average adsorption isotherm around the macroscopic jump, for various system sizes: $N=10,20$ and 25 (respectively $10^{5}, 10^{5}$, and $5 \times 10^{4}$ disorder realizations).

Figure 14: Quenched complexity $\sum_{\mathrm{Q}}^{\mathrm{L}-\mathrm{F}}(\rho ; \mu ; N)$ versus $\mu$ and $\rho$ for $N=30$. The colors are identical in both representations. The solid line in the right panel is the average main hysteresis loop for $N=30$. The average is performed for $5 \times 10^{3}$ disorder realizations. The density of metastable states vanishes for $\mu>-8.52$, even in the vicinity of the main adsorption branch (ellipse). 
Figure 1

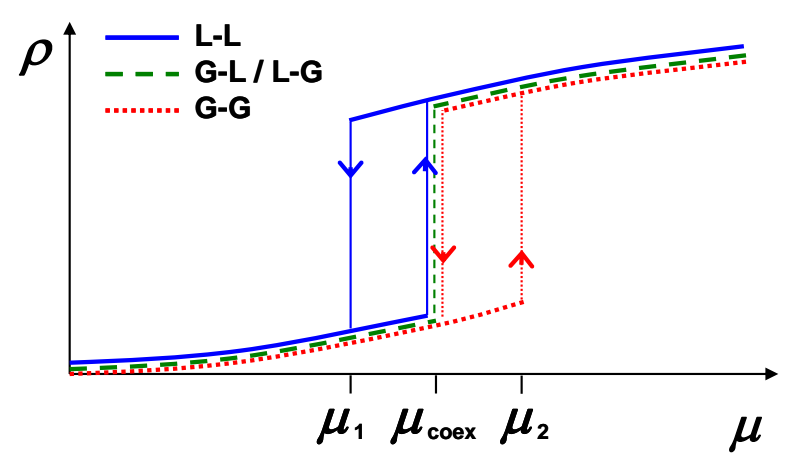


Figure 2

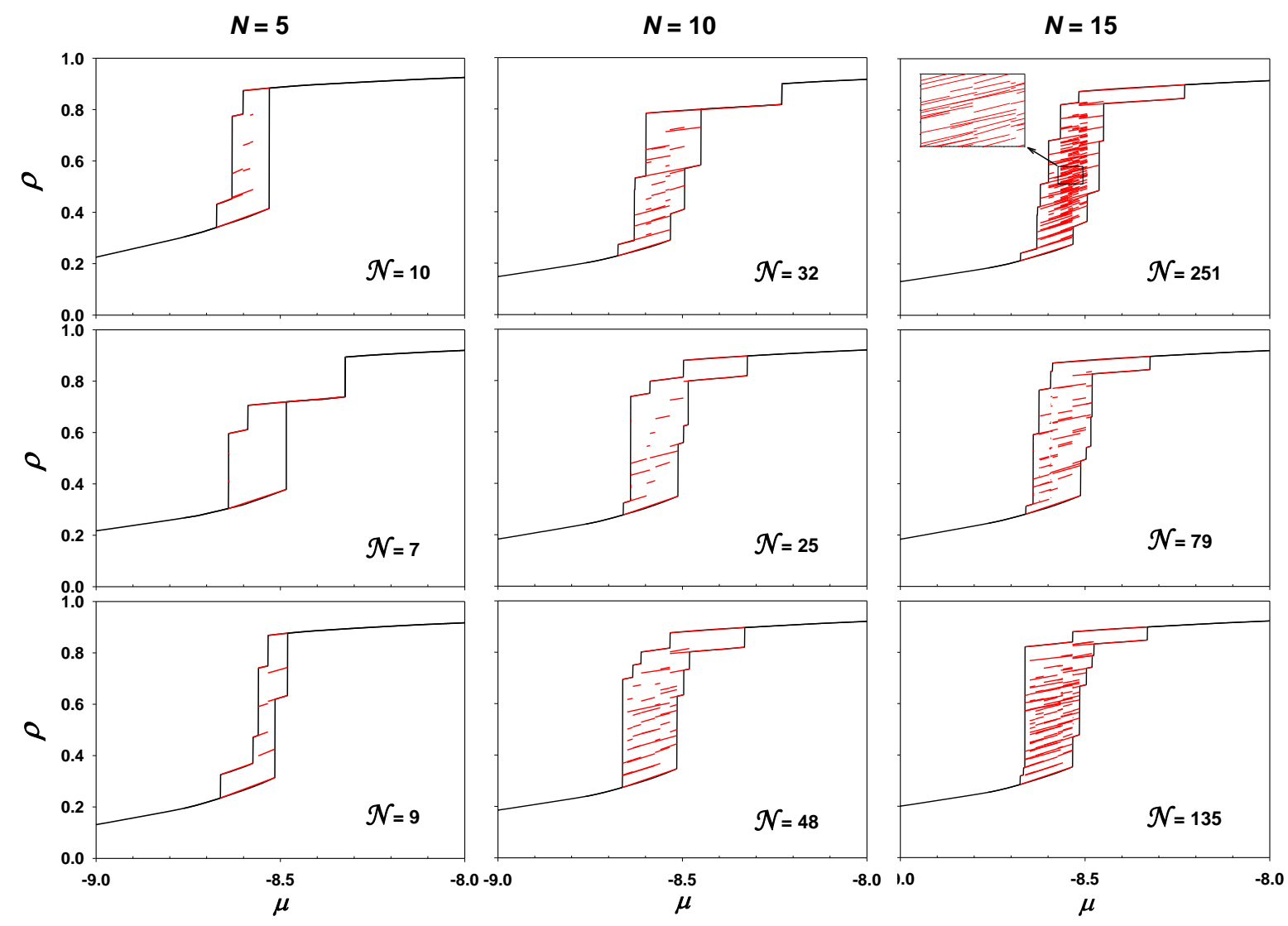


Figure 3
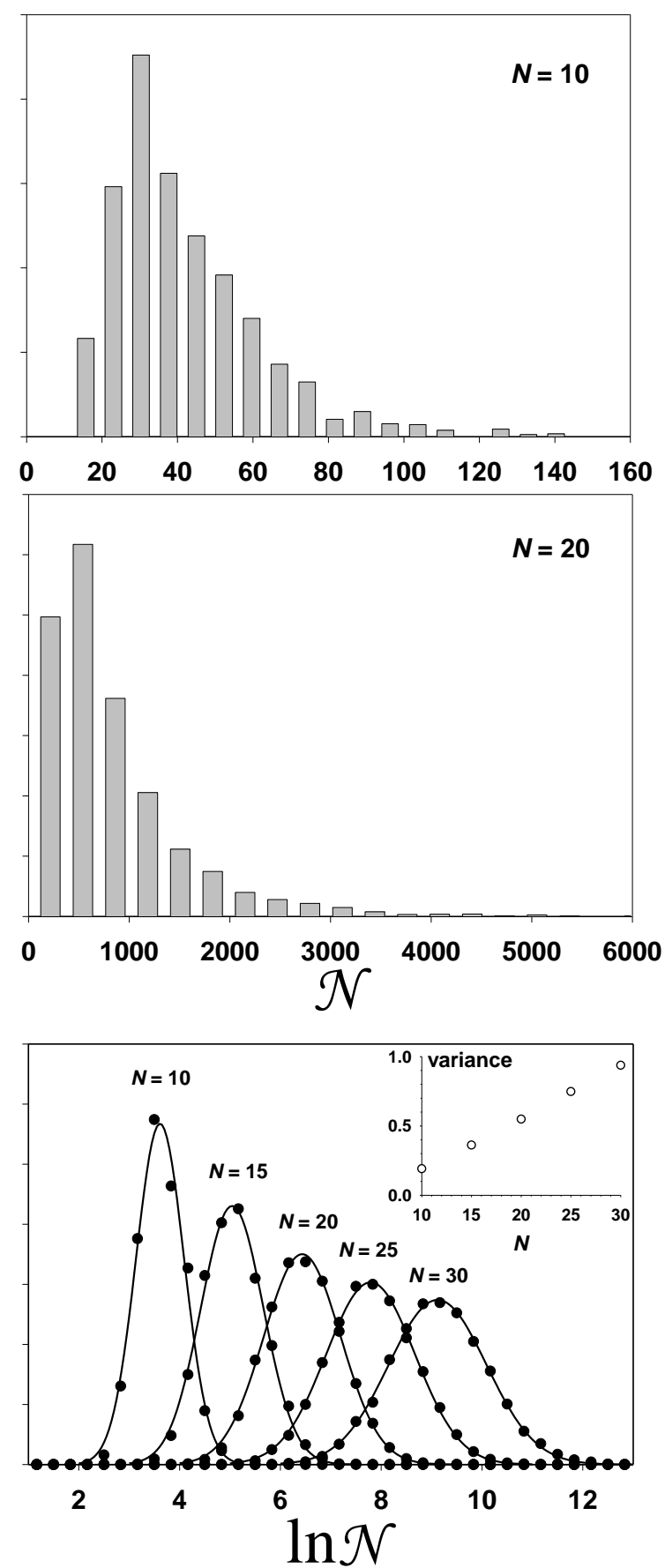
Figure 4

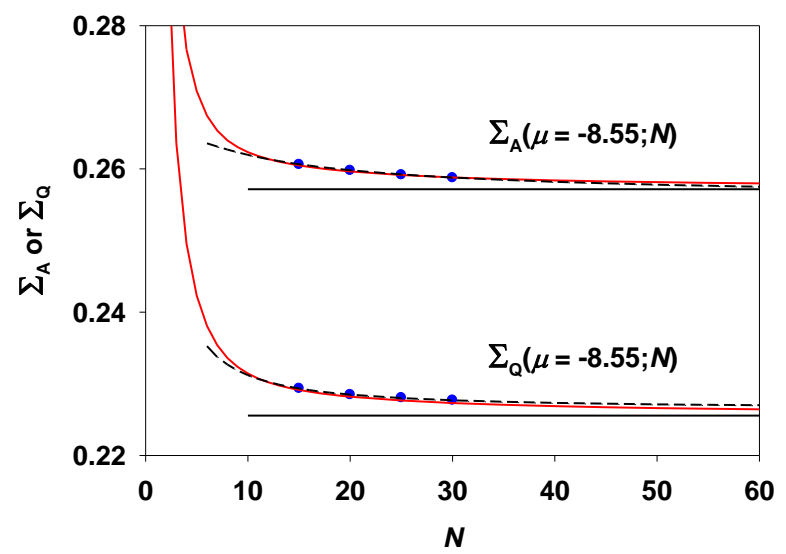


Figure 5

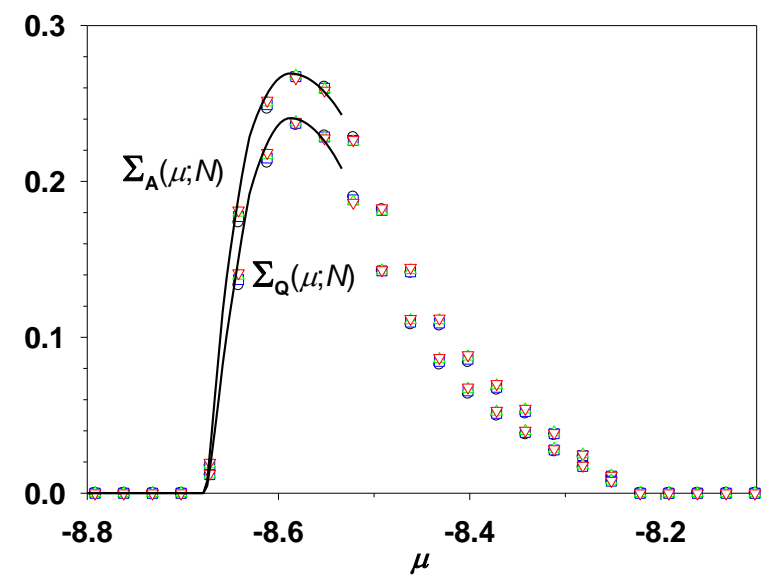


Figure 6
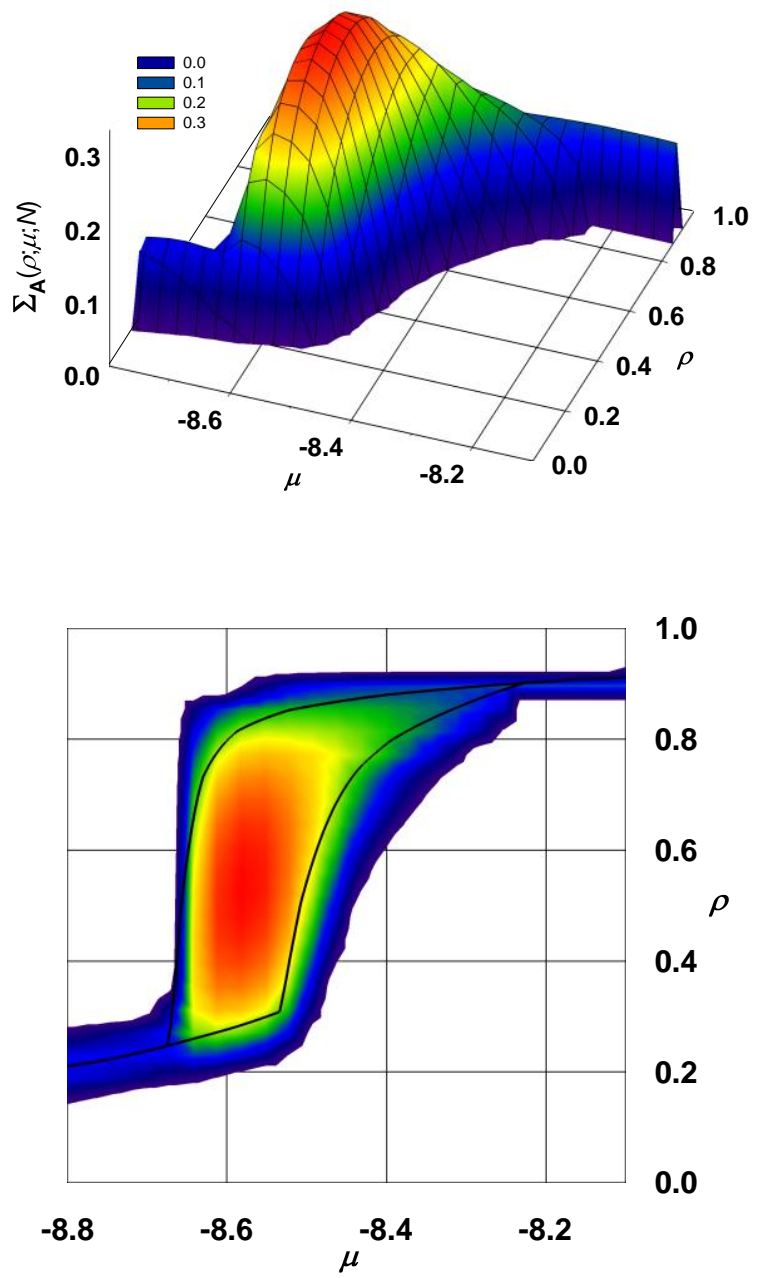
Figure 7

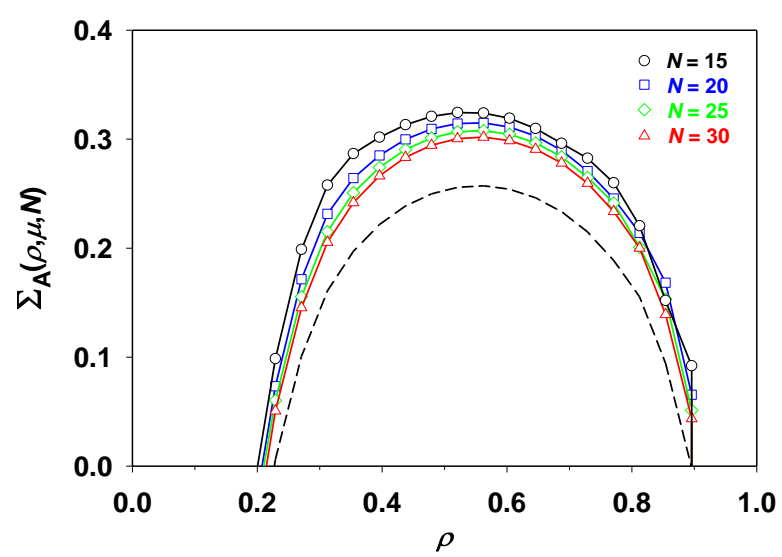


Figure 8
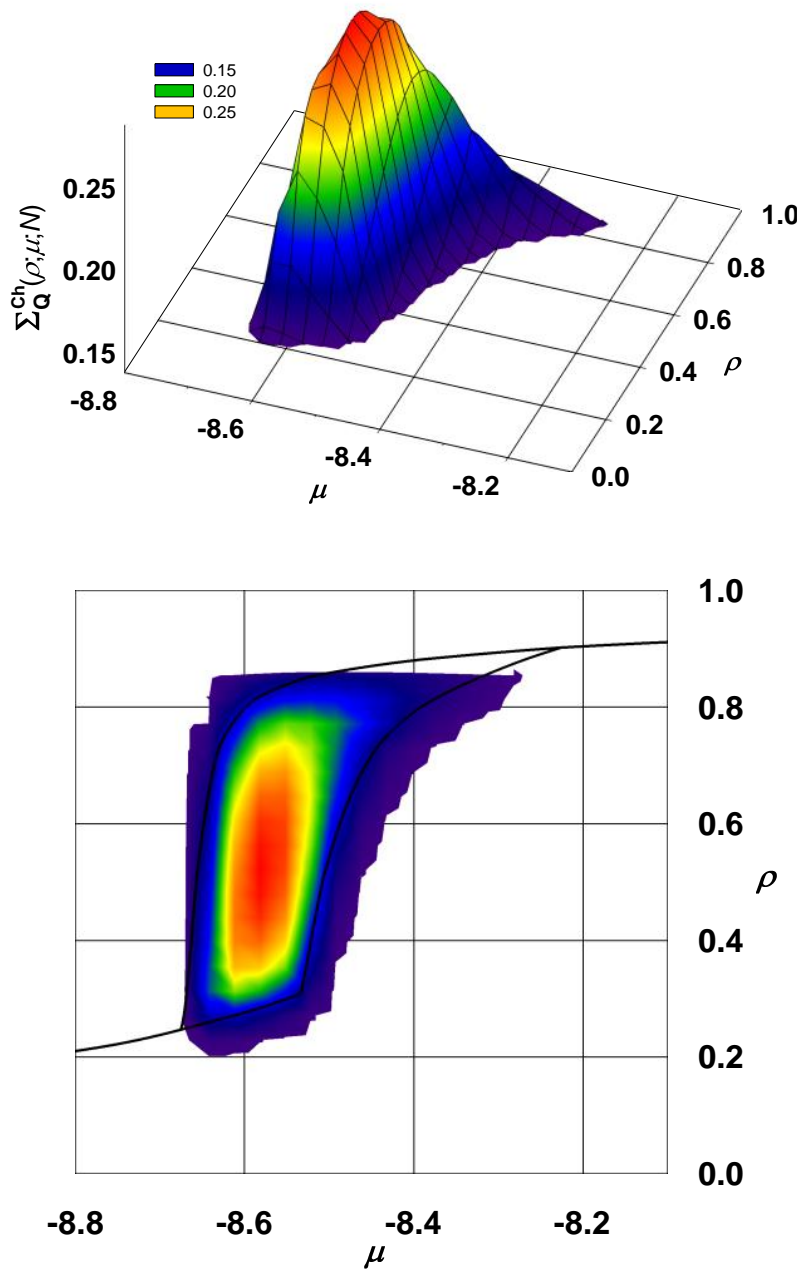
Figure 9

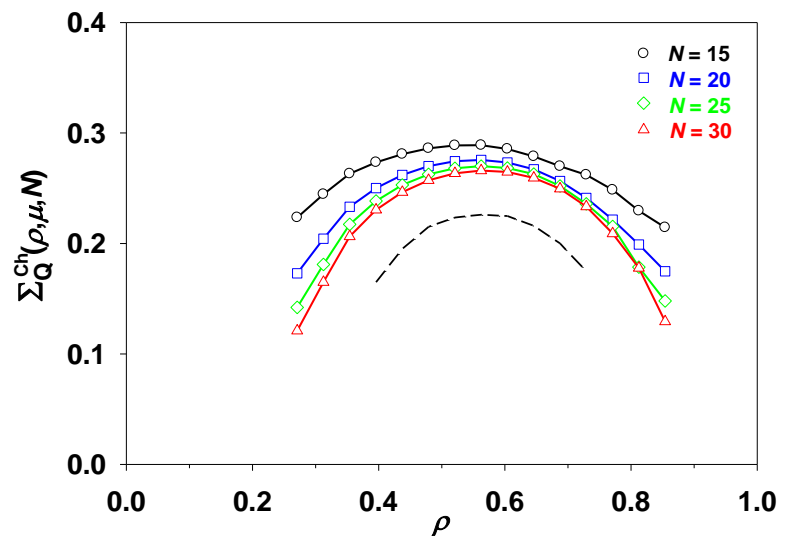


Figure 10
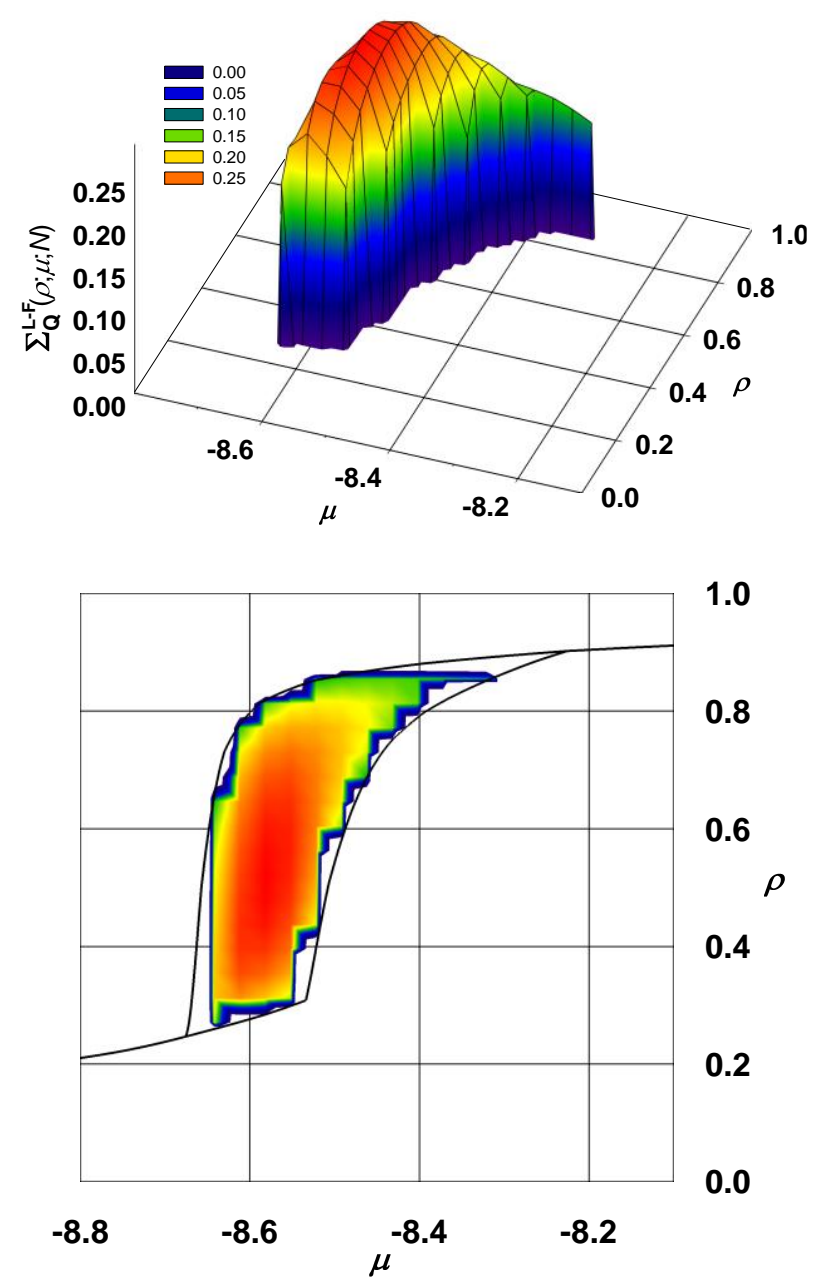
Figure 11

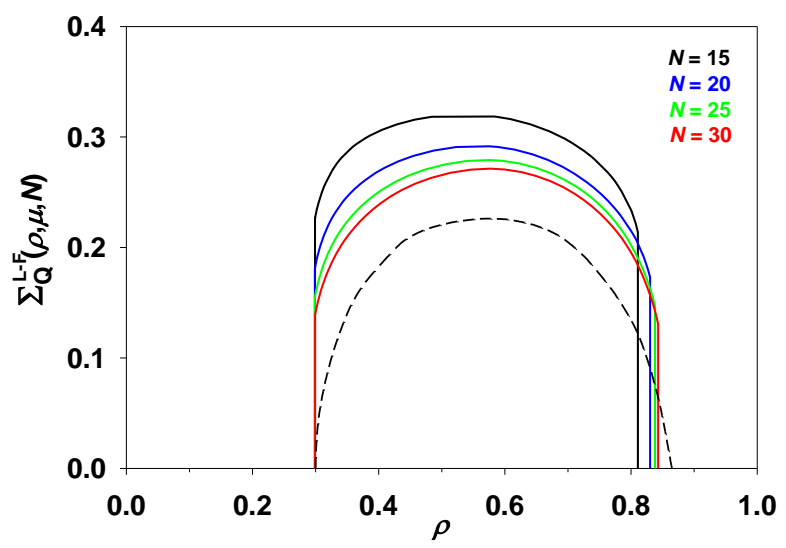


Figure 12

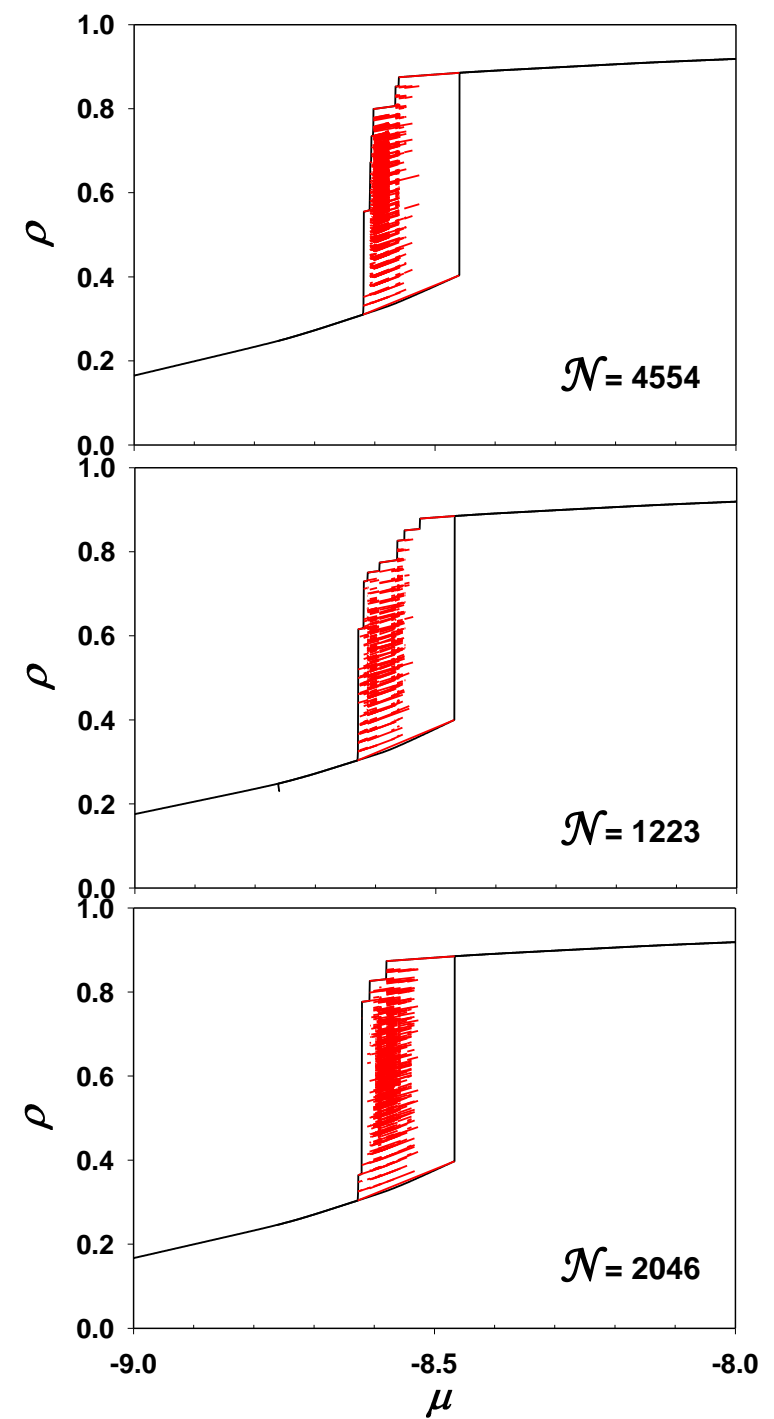


Figure 13

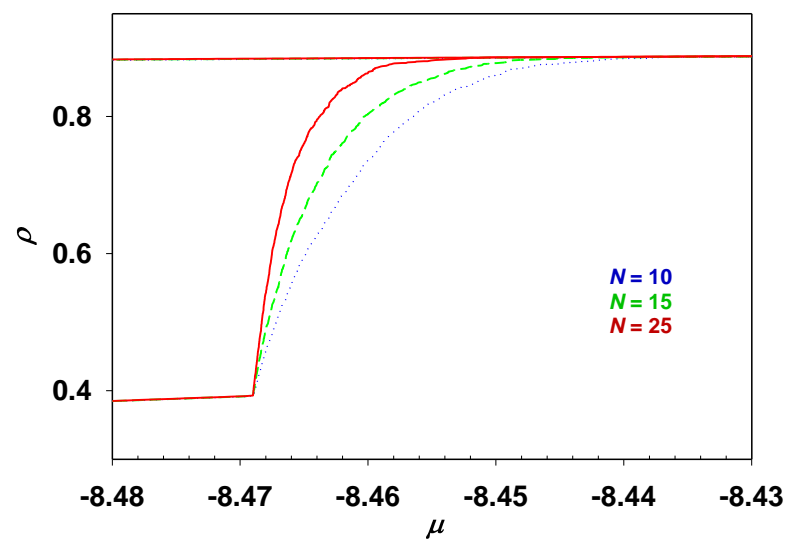


Figure 14
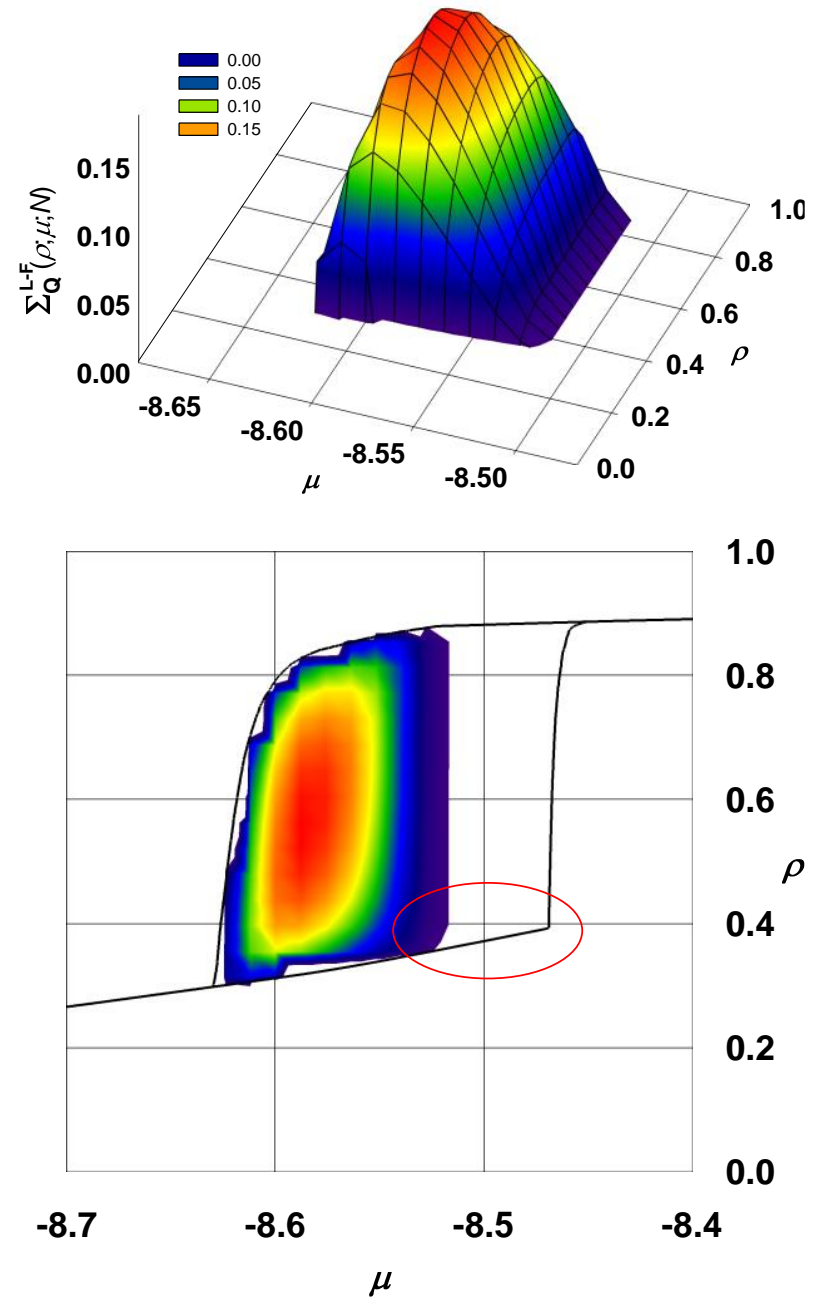